\title{
THE FIXED POINT SET OF A HOLOMORPHIC ISOMETRY, THE INTERSECTION OF TWO REAL FORMS IN A HERMITIAN SYMMETRIC SPACE OF COMPACT TYPE AND SYMMETRIC TRIADS
}

\author{
OSAMU IKAWA, MAKIKO SUMI TANAKA, AND HIROYUKI TASAKI
}

\begin{abstract}
We show a necessary and sufficient condition that the fixed point set of a holomorphic isometry and the intersection of two real forms of a Hermitian symmetric space of compact type are discrete and prove that they are antipodal sets in the cases. We also consider some relations between the intersection of two real forms and the fixed point set of a certain holomorphic isometry.
\end{abstract}

\section{INTRODUCTION}

In [15], [17] and [18] the second and third authors showed that the intersection of two real forms in a Hermitian symmetric space of compact type is an antipodal set if the intersection is discrete. The notion of an antipodal set of a Riemannian symmetric space was introduced by Chen-Nagano [3]. We showed the main results of this paper in a special case in [8]. In this paper we show a necessary and sufficient condition that the fixed point set of a holomorphic isometry of a Hermitian symmetric space of compact type is discrete and prove that the discrete fixed point set is an antipodal set. We also show a necessary and sufficient condition that the intersection of two real forms in a Hermitian symmetric space of compact type is discrete and consider some relations between the intersection of two real forms and the fixed point set of a certain holomorphic isometry by the use of the symmetric triads introduced by the first author in [6].

We roughly explain how to use symmetric triads in order to obtain a necessary and sufficient condition that the intersection of two real forms is discrete. In an irreducible Hermitian symmetric space $M=G / K$ of

The first author was partly supported by the Grant-in-Aid for Science Research (C) 2013 (No. 25400070), Japan Society for the Promotion of Science.

The second author was partly supported by the Grant-in-Aid for Science Research (C) 2013 (No. 23540108), Japan Society for the Promotion of Science.

The third author was partly supported by the Grant-in-Aid for Science Research (C) 2013 (No. 24540064), Japan Society for the Promotion of Science. 
compact type two real forms $L_{1}$ and $L_{2}$ are determined by two involutive anti-holomorphic isometries $\tau_{1}$ and $\tau_{2}$. These involutive isometries $\tau_{1}$ and $\tau_{2}$ determine two symmetric pairs $\left(G, F_{1}\right)$ and $\left(G, F_{2}\right)$. The triad $\left(G, F_{1}, F_{2}\right)$ defines a symmetric triad, by which we can describe the intersection $L_{1} \cap L_{2}$ and obtain a necessary and sufficient condition that $L_{1} \cap L_{2}$ is discrete.

The organization of this paper is as follows. In Section 2 we briefly review some fundamental results on Hermitian symmetric spaces of compact type, their antipodal sets and real forms.

In Section 3 we describe the fixed point set of a holomorphic isometry of a Hermitian symmetric space of compact type and obtain a necessary and sufficient condition that the fixed point set is discrete. If a holomorphic isometry is contained in the identity component of the group of holomorphic isometries, we can describe its fixed point set by the root system of the Lie algebra of the group of holomorphic isometries. There are two sequences of irreducible Hermitian symmetric spaces of compact type whose groups of holomorphic isometries are not connected. In these cases we describe the fixed point set of a holomorphic isometry which is not contained in the identity component in another way and obtain a necessary and sufficient condition that the fixed point set is discrete. In the cases where the fixed point sets are discrete, we describe them as orbits of certain Weyl groups.

In Section 4 we first describe a great antipodal set of each irreducible Hermitian symmetric space $M$ of compact type as an orbit of the Weyl group. We second investigate two real forms in $M$ and their intersection from a viewpoint of symmetric triads.

In Section 5 we also investigate a relation between the intersection of two real forms in $M$ and the fixed point set of a certain holomorphic isometry on $M$ from a viewpoint of symmetric triads.

The authors would like to thank Katsuya Mashimo and Kurando Baba for useful conversations. They are also indebted to the referee, whose comments improved the manuscript.

\section{Hermitian symmetric spaCes of COMPaCt type}

In this section we review some fundamental results on Hermitian symmetric spaces of compact type. We also review their antipodal sets and real forms which we need later.

We construct a Hermitian symmetric space of compact type as an adjoint orbit in a compact semisimple Lie algebra. Let $G$ be a connected compact semisimple Lie group and $\mathfrak{g}$ its Lie algebra, which is 
a compact semisimple Lie algebra. We take an $\operatorname{Ad}(G)$-invariant inner product $\langle$,$\rangle on \mathfrak{g}$. We take a nonzero element $J \in \mathfrak{g}$ satisfying $(\operatorname{ad} J)^{3}=-\operatorname{ad} J$. The adjoint orbit $M=\operatorname{Ad}(G) J \subset \mathfrak{g}$ is a Hermitian symmetric space of compact type with respect to the induced metric from $\langle$,$\rangle . Define a closed subgroup K$ of $G$ by

$$
K=\{k \in G \mid \operatorname{Ad}(k) J=J\} .
$$

Its Lie algebra $\mathfrak{k}$ is given by

$$
\mathfrak{k}=\{X \in \mathfrak{g} \mid[J, X]=0\} .
$$

The subspace

$$
\mathfrak{m}=\{[J, X] \mid X \in \mathfrak{g}\}
$$

is the orthogonal complement of $\mathfrak{k}$, thus we have an orthogonal direct sum decomposition $\mathfrak{g}=\mathfrak{k}+\mathfrak{m}$. The automorphism $e^{\pi \operatorname{ad} J}$ is involutive. The subalgebra $\mathfrak{k}$ is the $(+1)$-eigenspace and the subspace $\mathfrak{m}$ is the $(-1)$-eigenspace of $e^{\pi \mathrm{ad} J}$. The operator ad $J$ defines an $\operatorname{Ad}(K)$-invariant complex structure on $\mathfrak{m}$ which can be identified with the tangent space of $M$ at $J$, hence it defines an $\operatorname{Ad}(G)$-invariant complex structure on $M$. It is known that any Hermitian symmetric space of compact type is constructed in this manner.

In a Riemannian symmetric space $M$ we denote by $s_{x}$ the geodesic symmetry at $x \in M$. A subset $S$ of $M$ is an antipodal set if $s_{x}(y)=y$ for any $x, y \in S$. The 2-number $\#_{2} M$ of $M$ is the maximum of the cardinality of antipodal sets of $M$. We call an antipodal set of $M$ great if it attains $\#_{2} M$. These were introduced by Chen-Nagano [3].

A great antipodal set of a Hermitian symmetric space of compact type is described in the following way.

Theorem 2.1 ([16]). Let $M=\operatorname{Ad}(G) J \subset \mathfrak{g}$ be a Hermitian symmetric space of compact type. A great antipodal set of $M$ is represented as $M \cap \mathfrak{t}$ for a maximal abelian subalgebra $\mathfrak{t}$ of $\mathfrak{g}$ containing $J$. In particular, a great antipodal set of $M$ is an orbit of the Weyl group of $\mathfrak{g}$ with respect to $\mathrm{t}$.

After [16] was published we knew the following earlier results. Bott [1] showed that $M \cap \mathfrak{t}$ is an orbit of the Weyl group of $\mathfrak{g}$ and Takeuchi [13] showed that the Weyl group acts transitively on the great antipodal set of $M$.

Take a maximal abelian subalgebra $\mathfrak{t}$ of $\mathfrak{k}$. Since the involution $e^{\pi \mathrm{ad} J}$ is of inner type, $\mathfrak{t}$ is also a maximal abelian subalgebra of $\mathfrak{g}$. Since $J$ commutes any element of $\mathfrak{k}$, the maximality of $\mathfrak{t}$ implies that $J$ is in $\mathfrak{t}$. We will use the following lemma, which was suggested by K. Mashimo, in Subsection 4.1. 
Lemma 2.2. Denote by $W(\mathfrak{g})$ and $W(\mathfrak{k})$ the Weyl groups of $\mathfrak{g}$ and $\mathfrak{k}$ with respect to $\mathfrak{t}$ respectively. Then $W(\mathfrak{g}) J=W(\mathfrak{g}) / W(\mathfrak{k})$. In particular, $\#(W(\mathfrak{g}) J)=\#(W(\mathfrak{g})) / \#(W(\mathfrak{k}))$.

Proof. The isotropy subgroup of $W(\mathfrak{g})$ at $J$ is equal to

$$
\{s \in W(\mathfrak{g}) \mid s J=J\}=W(\mathfrak{g}) \cap \operatorname{Ad}_{G}(K)=W(\mathfrak{k}) .
$$

By definition, a real form is a fixed point set of an involutive antiholomorphic isometry of $M$. A real form of $M$ is a connected totally geodesic Lagrangian submanifold of $M$. Leung [9] and Takeuchi [12] classified real forms $L$ of irreducible Hermitian symmetric spaces $M$ of compact type. The list is as follows.

\begin{tabular}{|l|l|}
\hline$M$ & $L$ \\
\hline$G_{k}\left(\mathbb{C}^{n}\right)$ & $G_{k}\left(\mathbb{R}^{n}\right)$ \\
\hline$G_{2 k}\left(\mathbb{C}^{2 n}\right)$ & $G_{k}\left(\mathbb{H}^{n}\right)$ \\
\hline$G_{n}\left(\mathbb{C}^{2 n}\right)$ & $U(n)$ \\
\hline$Q_{n}(\mathbb{C})$ & $S^{k, n-k}$ \\
\hline$S O(4 n) / U(2 n)$ & $U(2 n) / S p(n)$ \\
\hline$S O(2 n) / U(n)$ & $S O(n)$ \\
\hline$S p(2 n) / U(2 n)$ & $S p(n)$ \\
\hline$S p(n) / U(n)$ & $U(n) / O(n)$ \\
\hline$E_{6} / S^{1} \cdot \operatorname{Spin}(10)$ & $G_{2}\left(\mathbb{H}^{4}\right) / \mathbb{Z}_{2}$ \\
\hline$E_{6} / S^{1} \cdot \operatorname{Spin}(10)$ & $P_{2}(\mathbb{C} a y)=F_{4} / \operatorname{Spin}(9)$ \\
\hline$E_{7} / S^{1} \cdot E_{6}$ & $(S U(8) / S p(4)) / \mathbb{Z}_{2}$ \\
\hline$E_{7} / S^{1} \cdot E_{6}$ & $S^{1} \cdot E_{6} / F_{4}$ \\
\hline
\end{tabular}

In this list above we denote by $G_{k}\left(\mathbb{K}^{n}\right)$ the Grassmann manifold consisting of $\mathbb{K}$-subspaces of $\mathbb{K}$-dimension $k$ in $\mathbb{K}^{n}$ for $\mathbb{K}=\mathbb{R}, \mathbb{C}, \mathbb{H}$ and by $Q_{n}(\mathbb{C})$ the complex hyperquadric of complex dimension $n$ in the complex projective space $\mathbb{C} P^{n+1}$, which is holomorphically isometric to the real oriented Grassmann manifold $\tilde{G}_{2}\left(\mathbb{R}^{n+2}\right)$. We regard $\tilde{G}_{2}\left(\mathbb{R}^{n+2}\right)$ as a submanifold in $\bigwedge^{2} \mathbb{R}^{n+2}$ in a natural way and define a real form $S^{p, q}$ of $\tilde{G}_{2}\left(\mathbb{R}^{n+2}\right)$ for $p, q$ with $p+q=n$ by

$$
S^{p, q}=S^{p}\left(\mathbb{R} e_{1} \oplus \cdots \oplus \mathbb{R} e_{p+1}\right) \wedge S^{q}\left(\mathbb{R} e_{p+2} \oplus \cdots \oplus \mathbb{R} e_{n+2}\right),
$$

where $e_{1}, \ldots, e_{n+2}$ is the standard orthonormal basis of $\mathbb{R}^{n+2}$. The real form $S^{p, q}$ is diffeomorphic to $\left(S^{p} \times S^{q}\right) / \mathbb{Z}_{2}$. 


\section{THE FIXED POINT SET OF A HOLOMORPHIC ISOMETRY}

In this section we show a necessary and sufficient condition that the fixed point set of a holomorphic isometry of a Hermitian symmetric space of compact type is discrete and prove that the discrete fixed point set is an antipodal set. We use the notation described in the last section.

For a set $X$ and a map $\phi: X \rightarrow X$ we denote

$$
F(\phi, X)=\{x \in X \mid \phi(x)=x\} .
$$

We use this notation throughout the paper. For any element $g$ in a connected compact semisimple Lie group $G$ with Lie algebra $\mathfrak{g}$ we have $\operatorname{dim} F(\operatorname{Ad}(g), \mathfrak{g}) \geq \operatorname{rank}(G)$, because there exists a maximal torus of $G$ containing $g$. If $\operatorname{dim} F(\operatorname{Ad}(g), \mathfrak{g})=\operatorname{rank}(G)$, we call $g$ a regular element of $G$. We can see that the set of all regular elements of $G$ is open and dense in $G$.

We denote by $A(M)$ the group of all holomorphic isometries of a Hermitian symmetric space $M$ of compact type and by $A_{0}(M)$ its identity component. If $M$ is equal to $\operatorname{Ad}(G) J \subset \mathfrak{g}$ for a connected compact semisimple Lie group $G$ with Lie algebra $\mathfrak{g}$, the identity component $A_{0}(M)$ coincides with $\left\{\left.\operatorname{Ad}(g)\right|_{M} \mid g \in G\right\}$. Without loss of generality we can suppose that the action of each simple factor of $G$ on $M$ is not trivial.

Theorem 3.1. Let $M=\operatorname{Ad}(G) J \subset \mathfrak{g}$ be a Hermitian symmetric space of compact type. The fixed point set $F(\operatorname{Ad}(g), M)$ is discrete if and only if $g$ is a regular element of $G$. In the case $F(\operatorname{Ad}(g), M)$ is a great antipodal set of $M$.

Proof. We take a maximal abelian subalgebra $\mathfrak{t}$ of $\mathfrak{g}$ containing $J$. Then we have $\mathfrak{t} \subset \mathfrak{k}$ by the definition of $\mathfrak{k}$. We denote by $\mathfrak{g}^{\mathbb{C}}$ the complexification of $\mathfrak{g}$. For $\alpha \in \mathfrak{t}$ we define the root space

$$
\mathfrak{g}_{\alpha}=\left\{X \in \mathfrak{g}^{\mathbb{C}} \mid[H, X]=\sqrt{-1}\langle\alpha, H\rangle X(H \in \mathfrak{t})\right\}
$$

and the root system $\Delta=\left\{\alpha \in \mathfrak{t}-\{0\} \mid \mathfrak{g}_{\alpha} \neq\{0\}\right\}$. Then we have the root space decomposition

$$
\mathfrak{g}^{\mathbb{C}}=\mathfrak{t}^{\mathbb{C}}+\sum_{\alpha \in \Delta} \mathfrak{g}_{\alpha}
$$

We define a lexicographic order on $\mathfrak{t}$ and write $\Delta_{+}=\{\alpha \in \Delta \mid \alpha>0\}$. We obtain

$$
\mathfrak{g}=\mathfrak{t}+\sum_{\alpha \in \Delta_{+}} \mathfrak{g} \cap\left(\mathfrak{g}_{\alpha}+\mathfrak{g}_{-\alpha}\right)
$$


We write $T=\exp \mathfrak{t}$, which is a maximal torus of $G$, hence there exists $g_{1} \in G$ such that $a=g_{1} g g_{1}^{-1} \in T$. Since

$$
\operatorname{Ad}\left(g_{1}\right) F(\operatorname{Ad}(g), M)=F(\operatorname{Ad}(a), M),
$$

we consider the condition that $F(\operatorname{Ad}(a), M)$ is discrete for $a \in T$. From the definition of $\mathfrak{g}_{\alpha}$ we obtain the following lemma.

Lemma 3.2. For $a=\exp H \in T$ with $H \in \mathfrak{t}$ we have

$$
F(\operatorname{Ad}(a), \mathfrak{g})=\mathfrak{t}+\sum_{\substack{\alpha \in \Delta,\langle\alpha, H\rangle \in 2 \pi \mathbb{Z}}} \mathfrak{g} \cap\left(\mathfrak{g}_{\alpha}+\mathfrak{g}_{-\alpha}\right) .
$$

Proof. By Lemma 3.1 of Chapter VI in Helgason [5] we can see that for $\alpha \in \Delta$ there exists a basis $F_{\alpha}, G_{\alpha}$ of $\mathfrak{g} \cap\left(\mathfrak{g}_{\alpha}+\mathfrak{g}_{-\alpha}\right)$ which satisfies

$$
\left[H, F_{\alpha}\right]=\langle\alpha, H\rangle G_{\alpha}, \quad\left[H, G_{\alpha}\right]=-\langle\alpha, H\rangle F_{\alpha} .
$$

These imply

$$
\begin{aligned}
& \operatorname{Ad}(\exp H) F_{\alpha}=\cos \langle\alpha, H\rangle F_{\alpha}+\sin \langle\alpha, H\rangle G_{\alpha}, \\
& \operatorname{Ad}(\exp H) G_{\alpha}=-\sin \langle\alpha, H\rangle F_{\alpha}+\cos \langle\alpha, H\rangle G_{\alpha} .
\end{aligned}
$$

Therefore we obtain

$$
F(\operatorname{Ad}(a), \mathfrak{g})=\mathfrak{t}+\sum_{\substack{\alpha \in \Delta,\langle\alpha, H\rangle \in 2 \pi \mathbb{Z}}} \mathfrak{g} \cap\left(\mathfrak{g}_{\alpha}+\mathfrak{g}_{-\alpha}\right) .
$$

Corollary 3.3. For $H \in \mathfrak{t}$ the following three conditions are equivalent.

(1) The element $\exp H$ is regular.

(2) $F(\operatorname{Ad}(\exp H), \mathfrak{g})=\mathfrak{t}$.

(3) $\langle\alpha, H\rangle \notin 2 \pi \mathbb{Z}$, for any $\alpha \in \Delta$.

Using these preliminaries we prove the theorem. We consider the case where $g$ is a regular element of $G$. In this case $a=g_{1} g g_{1}^{-1} \in T$ is also regular. By Corollary 3.3 we have $F(\operatorname{Ad}(a), \mathfrak{g})=\mathfrak{t}$ and hence

$$
F(\operatorname{Ad}(a), M)=M \cap \mathfrak{t},
$$

which is discrete and a great antipodal set of $M$.

Next we consider the case where $g$ is not a regular element. In this case $a=g_{1} g g_{1}^{-1} \in T$ is not regular. We write $a=\exp H$ for $H \in \mathfrak{t}$. By Corollary 3.3 there exists $\alpha \in \Delta$ which satisfies $\langle\alpha, H\rangle \in 2 \pi \mathbb{Z}$.

Let $\mathfrak{g}_{i}(1 \leq i \leq n)$ be simple ideals of $\mathfrak{g}$ and $\mathfrak{g}=\mathfrak{g}_{1} \oplus \cdots \oplus \mathfrak{g}_{n}$ is a direct sum decomposition of $\mathfrak{g}$. There exists $i$ such that $\alpha \in \mathfrak{t}_{i}=\mathfrak{t} \cap \mathfrak{g}_{i}$. We denote by $W_{i}$ the Weyl group of $\mathfrak{g}_{i}$ with respect to $\mathfrak{t}_{i}$ and by $J_{i}$ the $\mathfrak{g}_{i}$-component of $J$. Since the action of the Lie subgroup corresponding to $\mathfrak{g}_{i}$ is not trivial, we have $J_{i} \neq 0$. The Weyl group $W_{i}$ acts transitively 
on the set of all long roots of $\mathfrak{g}_{i}$ and the set of all short roots, thus we have

$$
\operatorname{span}_{\mathbb{R}}\left\{w \alpha \mid w \in W_{i}\right\}=\mathfrak{t}_{i}
$$

Hence there exists $w \in W_{i}$ which satisfies $\left\langle\alpha, w J_{i}\right\rangle \neq 0$. We can replace $J$ with $w J$. Then we have $\langle\alpha, J\rangle \neq 0$ and the decomposition

$$
J=\frac{\langle\alpha, J\rangle}{\langle\alpha, \alpha\rangle} \alpha+\left(J-\frac{\langle\alpha, J\rangle}{\langle\alpha, \alpha\rangle} \alpha\right)
$$

We take $F_{\alpha}$ in the proof of Lemma 3.2 and consider

$$
\begin{aligned}
\operatorname{Ad}\left(\exp t F_{\alpha}\right) J & =\operatorname{Ad}\left(\exp t F_{\alpha}\right) \frac{\langle\alpha, J\rangle}{\langle\alpha, \alpha\rangle} \alpha+\operatorname{Ad}\left(\exp t F_{\alpha}\right)\left(J-\frac{\langle\alpha, J\rangle}{\langle\alpha, \alpha\rangle} \alpha\right) \\
& =\operatorname{Ad}\left(\exp t F_{\alpha}\right) \frac{\langle\alpha, J\rangle}{\langle\alpha, \alpha\rangle} \alpha+\left(J-\frac{\langle\alpha, J\rangle}{\langle\alpha, \alpha\rangle} \alpha\right) .
\end{aligned}
$$

Since $\left[F_{\alpha}, G_{\alpha}\right]=\left\|F_{\alpha}\right\|^{2} \alpha=\left\|G_{\alpha}\right\|^{2} \alpha$, the first term is $\operatorname{Ad}\left(\exp t F_{\alpha}\right) \frac{\langle\alpha, J\rangle}{\langle\alpha, \alpha\rangle} \alpha=\cos \left(\left\|F_{\alpha}\right\|\|\alpha\| t\right) \frac{\langle\alpha, J\rangle}{\langle\alpha, \alpha\rangle} \alpha-\sin \left(\left\|F_{\alpha}\right\|\|\alpha\| t\right) \frac{\langle\alpha, J\rangle}{\left\|F_{\alpha}\right\|\|\alpha\|} G_{\alpha}$

and it is a circle in $\operatorname{span}_{\mathbb{R}}\left\{\alpha, G_{\alpha}\right\} \subset \mathfrak{t}+\mathfrak{g} \cap\left(\mathfrak{g}_{\alpha}+\mathfrak{g}_{-\alpha}\right)$. The second term is contained in $\mathfrak{t}$, hence we have $\operatorname{Ad}\left(\exp t F_{\alpha}\right) J \in F(\operatorname{Ad}(a), M)$, which means that $F(\operatorname{Ad}(a), M)$ is not discrete. Therefore $F(\operatorname{Ad}(g), M)$ is not discrete. This completes the proof of the theorem.

Remark 3.4. We note that $\langle\alpha, J\rangle=0, \pm 1$ for any $\alpha \in \Delta$ since $(\operatorname{ad} J)^{3}=-\operatorname{ad} J$. This fact will be used in Subsection 4.1.

We consider the fixed point set of an element of $A(M)-A_{0}(M)$. We recall the results on $A(M) / A_{0}(M)$ obtained by Takeuchi [11].

Lemma 3.5 ([11]). Let $M$ be an irreducible Hermitian symmetric space of compact type. Then $A(M) / A_{0}(M)$ are as follows.

(A) If $M=Q_{2 m}(\mathbb{C})(m \geq 2)$ or $M=G_{m}\left(\mathbb{C}^{2 m}\right)(m \geq 2)$, then $A(M) / A_{0}(M) \cong \mathbb{Z}_{2}$.

(B) Otherwise, $A(M)=A_{0}(M)$.

In the case where $M$ is irreducible, it is sufficient to consider the cases where $M=Q_{2 m}(\mathbb{C}), G_{m}\left(\mathbb{C}^{2 m}\right)(m \geq 2)$.

In the case where $M=Q_{2 m}(\mathbb{C})(m \geq 2)$, we can suppose that $G=$ $S O(2 m+2)$ and regard $M=S O(2 m+2) /(S O(2) \times S O(2 m))$ as a submanifold in $\bigwedge^{2} \mathbb{R}^{2 m+2}$ in a natural way. We take

$$
\phi=\left[\begin{array}{llll}
1 & & & \\
& \ddots & & \\
& & 1 & \\
& & & -1
\end{array}\right] \in O(2 m+2) .
$$


Then we have $A(M)=A_{0}(M) \cup \operatorname{Ad}(\phi) A_{0}(M)$ as we showed in the proof of Proposition 2.2 in [17]. Hence

$A(M)-A_{0}(M)=\operatorname{Ad}(\phi) A_{0}(M)=\operatorname{Ad}(\{g \in O(2 m+2) \mid \operatorname{det} g=-1\})$.

For any $g \in O(2 m+2)$ there exists $g_{1} \in O(2 m+2)$ which satisfies

$$
g_{1} g g_{1}^{-1}=\left[\begin{array}{ccccc}
R\left(\theta_{1}\right) & & & & \\
& \ddots & & & \\
& & R\left(\theta_{m}\right) & & \\
& & & 1 & \\
& & & & -1
\end{array}\right] \text {, }
$$

where

$$
R\left(\theta_{i}\right)=\left[\begin{array}{cc}
\cos \theta_{i} & -\sin \theta_{i} \\
\sin \theta_{i} & \cos \theta_{i}
\end{array}\right] \quad(1 \leq i \leq m) .
$$

Let $e_{i}$ be the $i$-th column vector of $g_{1}^{-1}$. Then $e_{1}, \ldots, e_{2 m+2}$ is an orthonormal basis of $\mathbb{R}^{2 m+2}$ and

$$
\begin{aligned}
& g\left[\begin{array}{ll}
e_{2 i-1} & e_{2 i}
\end{array}\right]=\left[\begin{array}{ll}
e_{2 i-1} & e_{2 i}
\end{array}\right] R\left(\theta_{i}\right) \quad(1 \leq i \leq m) \\
& g\left[\begin{array}{ll}
e_{2 m+1} & e_{2 m+2}
\end{array}\right]=\left[\begin{array}{ll}
e_{2 m+1} & e_{2 m+2}
\end{array}\right]\left[\begin{array}{cc}
1 & 0 \\
0 & -1
\end{array}\right] .
\end{aligned}
$$

Using these we can obtain the following theorem on the fixed point set of an element of $A(M)-A_{0}(M)$.

Theorem 3.6. Let $M=Q_{2 m}(\mathbb{C})(m \geq 2)$. Any element of $A(M)-$ $A_{0}(M)$ is equal to $\operatorname{Ad}(g)$ for $g \in O(2 m+2)$ with $\operatorname{det} g=-1 . F(\operatorname{Ad}(g), M)$ is discrete if and only if there exists $g_{1} \in O(2 m+2)$ such that (3.1) holds and that $R\left(\theta_{1}\right), \ldots, R\left(\theta_{m}\right)$ are different from each other. In the case

$$
F(\operatorname{Ad}(g), M)=W(S O(2 m+1)) J
$$

where $S O(2 m+1)$ is the stabilizer of $e_{2 m+2} . F(\operatorname{Ad}(g), M)$ is an antipodal set of $M$ and

$$
\# F(\operatorname{Ad}(g), M)=2 m<2 m+2=\#_{2} M .
$$

Proof. By (3.2) we have

$$
\left\{ \pm e_{1} \wedge e_{2}, \pm e_{3} \wedge e_{4}, \ldots, \pm e_{2 m-1} \wedge e_{2 m}\right\} \subset F(\operatorname{Ad}(g), M) .
$$

We suppose that there exist $i, j(i<j)$ satisfying $R\left(\theta_{i}\right)=R\left(\theta_{j}\right)$. For $\xi \in \mathbb{R}$ we write

$$
C(\xi)=\left[\begin{array}{cc}
\cos \xi & 0 \\
0 & \cos \xi
\end{array}\right], \quad S(\xi)=\left[\begin{array}{cc}
\sin \xi & 0 \\
0 & \sin \xi
\end{array}\right] .
$$


Then we have

$$
g\left[\begin{array}{llll}
e_{2 i-1} & e_{2 i} & e_{2 j-1} & e_{2 j}
\end{array}\right]\left[\begin{array}{c}
C(\xi) \\
S(\xi)
\end{array}\right]=\left[\begin{array}{llll}
e_{2 i-1} & e_{2 i} & e_{2 j-1} & e_{2 j}
\end{array}\right]\left[\begin{array}{c}
C(\xi) \\
S(\xi)
\end{array}\right] R\left(\theta_{i}\right),
$$

hence $g$ acts on the 2-dimensional subspace spanned by

$$
\left[\begin{array}{llll}
e_{2 i-1} & e_{2 i} & e_{2 j-1} & e_{2 j}
\end{array}\right]\left[\begin{array}{c}
C(\xi) \\
S(\xi)
\end{array}\right]
$$

as the rotation with angle $\theta_{i}$. Therefore

$$
\left(\cos \xi e_{2 i-1}+\sin \xi e_{2 j-1}\right) \wedge\left(\cos \xi e_{2 i}+\sin \xi e_{2 j}\right) \in F(\operatorname{Ad}(g), M)
$$

for any $\xi \in \mathbb{R}$ and $F(\operatorname{Ad}(g), M)$ is not discrete.

Next we suppose that $R\left(\theta_{i}\right)$ and $R\left(\theta_{j}\right)$ are different for any different $i, j$. Let $V_{i}$ be the 2-dimensional subspace spanned by $e_{2 i-1}$ and $e_{2 i}$. Then we have an orthogonal direct sum decomposition

$$
\mathbb{R}^{2 m+2}=V_{1} \oplus \cdots \oplus V_{m+1} .
$$

We take $u \wedge v \in F(\operatorname{Ad}(g), M)$ where $u$ and $v$ are orthonormal. We decompose $u$ and $v$ as follows.

$$
\begin{array}{ll}
u=u_{1}+\cdots+u_{m+1} & \left(u_{i} \in V_{i}, 1 \leq i \leq m+1\right) \\
v=v_{1}+\cdots+v_{m+1} & \left(v_{i} \in V_{i} 1 \leq i \leq m+1\right) .
\end{array}
$$

The element $g$ acts on these as follows.

$$
\begin{aligned}
& g u=R\left(\theta_{1}\right) u_{1}+\cdots+R\left(\theta_{m}\right) u_{m}+g u_{m+1} \\
& g v=R\left(\theta_{1}\right) v_{1}+\cdots+R\left(\theta_{m}\right) v_{m}+g v_{m+1} .
\end{aligned}
$$

On the other hand $u \wedge v \in F(\operatorname{Ad}(g), M)$, hence $g$ acts on the 2dimensional subspace spanned by $u$ and $v$ as a rotation. There exists $\xi \in \mathbb{R}$ which satisfies

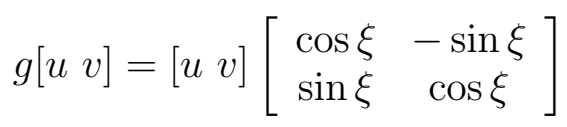

and we obtain

$$
\begin{aligned}
& g u=\cos \xi u+\sin \xi v \\
& g v=-\sin \xi u+\cos \xi v .
\end{aligned}
$$

Thus we get

$$
\begin{aligned}
& R\left(\theta_{i}\right) u_{i}=\cos \xi u_{i}+\sin \xi v_{i} \quad(1 \leq i \leq m), \\
& g u_{m+1}=\cos \xi u_{m+1}+\sin \xi v_{m+1} .
\end{aligned}
$$

The action of $g$ on $V_{m+1}$ is not a rotation, we have $u_{m+1}=0$. Since $R\left(\theta_{i}\right) \neq R\left(\theta_{j}\right)$ for different $i, j$, there exists $k$ such that

$$
u_{k} \neq 0, \quad u_{i}=0 \quad(i \neq k)
$$


and we have $u \wedge v= \pm e_{2 k-1} \wedge e_{2 k}$. Therefore

$$
F(\operatorname{Ad}(g), M)=\left\{ \pm e_{1} \wedge e_{2}, \pm e_{3} \wedge e_{4}, \ldots, \pm e_{2 m-1} \wedge e_{2 m}\right\}
$$

and $F(\operatorname{Ad}(g), M)$ is discrete. The above description of $F(\operatorname{Ad}(g), M)$ shows that it is an orbit of $W(S O(2 m+1))$ through $J=e_{1} \wedge e_{2}$ and an antipodal set of $M$.

In the case where $M=G_{m}\left(\mathbb{C}^{2 m}\right)(m \geq 2)$, we can suppose that $G=S U(2 m)$. We take

$$
J_{m}=\left[\begin{array}{rr} 
& 1_{m} \\
-1_{m} &
\end{array}\right]
$$

in $G$, where $1_{m}$ denotes the $m \times m$ identity matrix. We regard $M$ as the submanifold $\operatorname{Ad}(G) J$ in $\mathfrak{g}=\mathfrak{s u}(2 m)$, where

$$
J=\frac{\sqrt{-1}}{2}\left[\begin{array}{ll}
1_{m} & \\
& -1_{m}
\end{array}\right] \in \mathfrak{g} .
$$

We define an involutive automorphism $\phi$ of $G$ by $\phi(g)=J_{m} \bar{g} J_{m}^{-1}$ for $g \in G$. The fixed point set $F(\phi, G)$ is equal to $S p(m)$ and $\phi$ defines a symmetric pair $(S U(2 m), S p(m))$. The differential map $d \phi$ of $\phi$ is represented by $d \phi(X)=J_{m} \bar{X} J_{m}^{-1}$ for $X \in \mathfrak{g}$. So we simply write $\phi(X)=J_{m} \bar{X} J_{m}^{-1}$ for $X \in \mathfrak{g}$. The automorphism $\phi$ of $G$ also induces a holomorphic isometry of $M$ defined by

$$
\operatorname{Ad}(g) J \mapsto \operatorname{Ad}(\phi(g)) J \quad(g \in G) .
$$

Since $\phi(J)=J$, we have

$$
\operatorname{Ad}(\phi(g)) J=\operatorname{Ad}(\phi(g)) \phi(J)=\phi(\operatorname{Ad}(g) J) .
$$

Thus the holomorphic isometry of $M$ induced by $\phi$ is the restriction of $\phi: \mathfrak{g} \rightarrow \mathfrak{g}$ to $M \subset \mathfrak{g}$. So we also denote it by $\phi$.

The holomorphic isometry $\phi$ is contained in $A(M)-A_{0}(M)$, which is showed in [17]. We take a maximal torus $T$ of $S p(m)$.

Lemma 3.7. For any $h \in A(M)-A_{0}(M)$ there exist $t \in T$ and $g \in G$ such that $h=\operatorname{Ad}(g) \operatorname{Ad}(t) \operatorname{Ad}\left(\phi\left(g^{-1}\right)\right) \phi$ and

$$
F(h, M)=\operatorname{Ad}(g) F(\operatorname{Ad}(t) \phi, M) .
$$

Proof. We define two involutive automorphisms $\theta_{1}, \theta_{2}$ of $G \times G$ by

$$
\theta_{1}(g, h)=(h, g), \quad \theta_{2}(g, h)=\left(\phi^{-1}(h), \phi(g)\right) \quad((g, h) \in G \times G) .
$$

For a general automorphism $\phi$ of $G$, the automorphism $\theta_{2}$ is involutive. Since $\phi$ is involutive, $\theta_{1}$ and $\theta_{2}$ are commutative. The fixed point sets 
of them are

$$
\begin{aligned}
& K_{1}=F\left(\theta_{1}, G \times G\right)=\{(g, g) \mid g \in G\}, \\
& K_{2}=F\left(\theta_{2}, G \times G\right)=\{(g, \phi(g)) \mid g \in G\}
\end{aligned}
$$

and we obtain two direct sum decompositions of $\mathfrak{g} \times \mathfrak{g}$ as follows

$$
\mathfrak{g} \times \mathfrak{g}=\mathfrak{k}_{1} \oplus \mathfrak{m}_{1}=\mathfrak{k}_{2} \oplus \mathfrak{m}_{2},
$$

where

$$
\begin{aligned}
& \mathfrak{k}_{1}=\{(X, X) \mid X \in \mathfrak{g}\}, \quad \mathfrak{m}_{1}=\{(X,-X) \mid X \in \mathfrak{g}\}, \\
& \mathfrak{k}_{2}=\{(X, \phi(X)) \mid X \in \mathfrak{g}\}, \quad \mathfrak{m}_{2}=\{(X,-\phi(X)) \mid X \in \mathfrak{g}\} .
\end{aligned}
$$

These imply

$$
\begin{aligned}
& \mathfrak{k}_{1} \cap \mathfrak{k}_{2}=\{(X, X) \mid X \in \mathfrak{s p}(m)\}, \\
& \mathfrak{m}_{1} \cap \mathfrak{m}_{2}=\{(X,-X) \mid X \in \mathfrak{s p}(m)\} .
\end{aligned}
$$

We denote by $\mathfrak{t}$ the Lie algebra of $T$. The subspace

$$
\mathfrak{a}=\{(H,-H) \mid H \in \mathfrak{t}\}
$$

is a maximal abelian subspace of $\mathfrak{m}_{1} \cap \mathfrak{m}_{2}$. We identify $(g, \phi(g)) \in K_{2}$ with $g \in G$ and $\left(g_{1}, g_{2}\right) K_{1} \in(G \times G) / K_{1}$ with $g_{1} g_{2}^{-1} \in G$. Then the action of $K_{2}$ on $(G \times G) / K_{1}$ is equivalent with the action of $G$ on $G$ defined by

$$
g \cdot x=g x \phi\left(g^{-1}\right) \quad(g, x \in G) .
$$

This action is a Hermann action and $\theta_{1}, \theta_{2}$ are commutative. In particular the action is a hyperpolar action with section $T$ by [4]. So we have

$$
G=\bigcup_{g \in G} g T \phi\left(g^{-1}\right) .
$$

Since $A(M)=A_{0}(M) \cup A_{0}(M) \phi$, for any $h \in A(M)-A_{0}(M)$ there exist $g \in G$ and $t \in T$ such that

$$
h=\operatorname{Ad}(g) \operatorname{Ad}(t) \operatorname{Ad}\left(\phi\left(g^{-1}\right)\right) \phi .
$$

Using this we get

$$
F(h, M)=\left\{X \in M \mid \operatorname{Ad}(g) \operatorname{Ad}(t) \operatorname{Ad}\left(\phi\left(g^{-1}\right)\right) \phi(X)=X\right\} .
$$

We put $X=\operatorname{Ad}(g) Y$. Since $\phi(\operatorname{Ad}(g) Y)=\operatorname{Ad}(\phi(g)) \phi Y$, we obtain

$$
F(h, M)=\operatorname{Ad}(g) F(\operatorname{Ad}(t) \phi, M) .
$$


The above lemma shows that it is sufficient to consider the fixed point set $F(\operatorname{Ad}(t) \phi, M)$ for $t \in T$. We take a maximal torus

$$
T=\left\{\left[\begin{array}{ll}
Z & \\
& \bar{Z}
\end{array}\right] \mid Z \in U(1)^{m}\right\}
$$

of $S p(m)$ in order to calculate $F(\operatorname{Ad}(t) \phi, M)$. The Lie algebra of $T$ is

$$
\mathfrak{t}=\left\{H\left(x_{1}, \ldots, x_{m}\right) \mid x_{j} \in \mathbb{R}\right\},
$$

where

$$
H\left(x_{1}, \ldots, x_{m}\right)=\sqrt{-1}\left[\begin{array}{cccccc}
x_{1} & & & & & \\
& \ddots & & & & \\
& & x_{m} & & & \\
& & -x_{1} & & \\
& & & \ddots & \\
& & & & & -x_{m}
\end{array}\right] .
$$

We define $e_{i} \in \mathfrak{t}$ by

$$
\left\langle H\left(x_{1}, \ldots, x_{m}\right), e_{i}\right\rangle=x_{i} .
$$

The canonical decomposition $\mathfrak{g}=\mathfrak{k} \oplus \mathfrak{m}$ of the compact symmetric pair $(S U(2 m), S p(m))$ is given by

$$
\begin{aligned}
& \mathfrak{k}=\mathfrak{s p}(m)=\left\{\left[\begin{array}{cc}
X & -\bar{Y} \\
Y & \bar{X}
\end{array}\right] \mid \begin{array}{l}
X \in \mathfrak{u}(m), \\
{ }^{t} Y=Y \in \mathfrak{g l}(m, \mathbb{C})
\end{array}\right\}, \\
& \mathfrak{m}=\left\{\left[\begin{array}{cc}
X & \bar{Y} \\
Y & { }^{t} X
\end{array}\right] \mid \begin{array}{l}
X \in \mathfrak{u}(m), \\
-{ }^{t} Y=Y \in \mathfrak{g l}(m, \mathbb{C})
\end{array}\right\} .
\end{aligned}
$$

In order to consider the action of $\operatorname{Ad}(t) \phi$ on $\mathfrak{g}$ we first decompose $\mathfrak{k}$ into a direct sum of root spaces with respect to $\mathfrak{t}$. We define

$$
F_{i j}^{-}=\left[\begin{array}{cc}
E_{i j}-E_{j i} & \\
& E_{i j}-E_{j i}
\end{array}\right], G_{i j}^{-}=\sqrt{-1}\left[\begin{array}{ll}
E_{i j}+E_{j i} & \\
& -\left(E_{i j}+E_{j i}\right)
\end{array}\right]
$$

for $1 \leq i<j \leq m$ and

$$
F_{i j}^{+}=\left[\begin{array}{cc}
-\left(E_{i j}+E_{j i}\right) & E_{i j}+E_{j i}
\end{array}\right], G_{i j}^{+}=\sqrt{-1}\left[\begin{array}{cc}
E_{i j}+E_{j i} & E_{j i}
\end{array}\right]
$$

for $1 \leq i \leq j \leq m$. We get

$$
\mathfrak{s p}(m)=\mathfrak{t} \oplus \sum_{i<j}\left(\mathbb{R} F_{i j}^{-} \oplus \mathbb{R} G_{i j}^{-}\right) \oplus \sum_{i \leq j}\left(\mathbb{R} F_{i j}^{+} \oplus \mathbb{R} G_{i j}^{+}\right)
$$

and

$$
\left[H, F_{i j}^{ \pm}\right]=\left\langle e_{i} \pm e_{j}, H\right\rangle G_{i j}^{ \pm}, \quad\left[H, G_{i j}^{ \pm}\right]=-\left\langle e_{i} \pm e_{j}, H\right\rangle F_{i j}^{ \pm}
$$


for any $H \in \mathfrak{t}$. We second decompose $\mathfrak{m}$ into a direct sum of weight spaces with respect to $t$. We define

$$
\begin{aligned}
& X_{i j}^{-}=\left[\begin{array}{ll}
E_{i j}-E_{j i} & -\left(E_{i j}-E_{j i}\right)
\end{array}\right], Y_{i j}^{-}=\sqrt{-1}\left[\begin{array}{ll}
E_{i j}+E_{j i} & E_{i j}+E_{j i}
\end{array}\right], \\
& X_{i j}^{+}=\left[\begin{array}{ll}
E_{i j}-E_{j i} & E_{j i} \\
E_{i j}-E_{j i}
\end{array}\right], Y_{i j}^{+}=\sqrt{-1}\left[\begin{array}{cc}
-\left(E_{i j}-E_{j i}\right) &
\end{array}\right]
\end{aligned}
$$

for $1 \leq i<j \leq m$ and

$$
V(\mathfrak{m})=\{X \in \mathfrak{m} \mid[X, \mathfrak{t}]=\{0\}\} .
$$

We get

$$
\mathfrak{m}=V(\mathfrak{m}) \oplus \sum_{i<j}\left(\mathbb{R} X_{i j}^{-} \oplus \mathbb{R} Y_{i j}^{-}\right) \oplus \sum_{i<j}\left(\mathbb{R} X_{i j}^{+} \oplus \mathbb{R} Y_{i j}^{+}\right)
$$

and

$$
\left[H, X_{i j}^{ \pm}\right]=\left\langle e_{i} \pm e_{j}, H\right\rangle Y_{i j}^{ \pm}, \quad\left[H, Y_{i j}^{ \pm}\right]=-\left\langle e_{i} \pm e_{j}, H\right\rangle X_{i j}^{ \pm}
$$

for any $H \in \mathfrak{t}$. Using the above decompositions we obtain

(3.3) $F(\operatorname{Ad}(\exp H) \circ \phi, \mathfrak{g})$

$$
\begin{aligned}
& =\mathfrak{t} \oplus \sum_{\left\langle e_{i}+e_{j}, H\right\rangle \in 2 \pi \mathbb{Z}}\left(\mathbb{R} F_{i j}^{+} \oplus \mathbb{R} G_{i j}^{+}\right) \oplus \sum_{\left\langle e_{i}-e_{j}, H\right\rangle \in 2 \pi \mathbb{Z}}\left(\mathbb{R} F_{i j}^{-} \oplus \mathbb{R} G_{i j}^{-}\right) \\
& \oplus \sum_{\left\langle e_{i}+e_{j}, H\right\rangle \in \pi+2 \pi \mathbb{Z}}\left(\mathbb{R} X_{i j}^{+} \oplus \mathbb{R} Y_{i j}^{+}\right) \oplus \sum_{\left\langle e_{i}-e_{j}, H\right\rangle \in \pi+2 \pi \mathbb{Z}}\left(\mathbb{R} X_{i j}^{-} \oplus \mathbb{R} Y_{i j}^{-}\right)
\end{aligned}
$$

and the following theorem.

Theorem 3.8. Let $M=G_{m}\left(\mathbb{C}^{2 m}\right)(m \geq 2)$. Any element $h$ of $A(M)-$ $A_{0}(M)$ is equal to $\operatorname{Ad}(g) \operatorname{Ad}(\exp H) \operatorname{Ad}\left(\phi\left(g^{-1}\right)\right) \phi$ for $g \in S U(2 m), H \in$ t. Its fixed point set $F(h, M)$ is discrete if and only if $\left\langle e_{i} \pm e_{j}, H\right\rangle \notin \pi \mathbb{Z}$ for any $i \neq j$ and $\left\langle e_{i}, H\right\rangle \notin \pi \mathbb{Z}$ for any $i$. In the case

$$
F(\operatorname{Ad}(\exp H) \circ \phi, M)=W(S p(m)) J,
$$

which is an antipodal set of $M$ and

$$
\# F(h, M)=2^{m}<\left(\begin{array}{c}
2 m \\
m
\end{array}\right)=\#_{2} M .
$$

Proof. Lemma 3.7 implies the description of $h$ and it is sufficient to consider $F(\operatorname{Ad}(\exp H) \phi, M)$. According to $(3.3), F(\operatorname{Ad}(\exp H) \phi, \mathfrak{g})=$ $\mathfrak{t}$ if and only if $\left\langle e_{i} \pm e_{j}, H\right\rangle \notin \pi \mathbb{Z}$ for any $i \neq j$ and $\left\langle e_{i}, H\right\rangle \notin \pi \mathbb{Z}$ for any $i$. In this case we have

$$
F(\operatorname{Ad}(\exp H) \circ \phi, M)=M \cap \mathfrak{t}=W(S p(m)) J
$$

and $\# F(h, M)=2^{m}$. 
In order to prove the theorem we have to show that $F(\operatorname{Ad}(\exp H) \phi, M)$ is not discrete if $F(\operatorname{Ad}(\exp H) \phi, \mathfrak{g}) \neq \mathfrak{t}$. We first consider the case where there exist $i, j$ such that $\left\langle e_{i}+e_{j}, H\right\rangle \in 2 \pi \mathbb{Z}$. Since $W(S p(m)) J$ spans $\mathfrak{t}$, there exists $X \in W(S p(m)) J$ satisfying $\left\langle e_{i}+e_{j}, X\right\rangle \neq 0$. Using $\left[F_{i j}^{+}, G_{i j}^{+}\right]=\left|F_{i j}^{+}\right|^{2}\left(e_{i}+e_{j}\right)$ obtained from their definitions, we get

$$
\begin{aligned}
& \operatorname{Ad}\left(\exp t F_{i j}^{+}\right) X=\left(X-\frac{\left\langle e_{i}+e_{j}, X\right\rangle}{\left\|e_{i}+e_{j}\right\|^{2}}\left(e_{i}+e_{j}\right)\right) \\
& +\frac{\left\langle e_{i}+e_{j}, X\right\rangle}{\left\|e_{i}+e_{j}\right\|}\left(\cos \left(\left\|F_{i j}^{+}\right\|\left\|e_{i}+e_{j}\right\| t\right) \frac{e_{i}+e_{j}}{\left\|e_{i}+e_{j}\right\|}-\sin \left(\left\|F_{i j}^{+}\right\|\left\|e_{i}+e_{j}\right\| t\right) \frac{G_{i j}^{+}}{\left\|G_{i j}^{+}\right\|}\right)
\end{aligned}
$$

for $t \in \mathbb{R}$. Therefore $\operatorname{Ad}\left(\exp t F_{i j}^{+}\right) X \in F(\operatorname{Ad}(\exp H) \circ \phi, M)$ for $t \in \mathbb{R}$ and $F(\operatorname{Ad}(\exp H) \circ \phi, M)$ is not discrete. We second consider the case where there exist $i \neq j$ such that $\left\langle e_{i}-e_{j}, H\right\rangle \in \pi+2 \pi \mathbb{Z}$. There exists $X \in W(S p(m)) J$ satisfying $\left\langle e_{i}-e_{j}, X\right\rangle \neq 0$. Using $\left[X_{i j}^{-}, Y_{i j}^{-}\right]=$ $\left\|X_{i j}^{-}\right\|^{2}\left(e_{i}-e_{j}\right)$ obtained from their definitions, we get

$$
\begin{aligned}
& \operatorname{Ad}\left(\exp t X_{i j}^{-}\right) X=\left(X-\frac{\left\langle e_{i}-e_{j}, X\right\rangle}{\left\|e_{i}-e_{j}\right\|^{2}}\left(e_{i}-e_{j}\right)\right) \\
& +\frac{\left\langle e_{i}-e_{j}, X\right\rangle}{\left\|e_{i}-e_{j}\right\|}\left(\cos \left(\left\|X_{i j}^{-}\right\|\left\|e_{i}-e_{j}\right\| t\right) \frac{e_{i}-e_{j}}{\left\|e_{i}-e_{j}\right\|}-\sin \left(\left\|X_{i j}^{-}\right\|\left\|e_{i}-e_{j}\right\| t\right) \frac{Y_{i j}^{-}}{\left\|Y_{i j}^{-}\right\|}\right)
\end{aligned}
$$

for $t \in \mathbb{R}$. Therefore $\operatorname{Ad}\left(\exp t X_{i j}^{-}\right) X \in F(\operatorname{Ad}(\exp H) \circ \phi, M)$ for $t \in \mathbb{R}$ and $F(\operatorname{Ad}(\exp H) \circ \phi, M)$ is not discrete. In the other cases we can see that $F(\operatorname{Ad}(\exp H) \circ \phi, M)$ is not discrete in a similar way. This completes the proof of the theorem.

Remark 3.9. When a Hermitian symmetric space $M$ of compact type is not irreducible, $F(h, M)$ for $h \in A(M)$ is obtained from the fixed point sets of holomorphic isometries of irreducible Hermitian symmetric spaces of compact type, which we have already known above. Let $M=M_{1} \times \cdots \times M_{k}$ be the decomposition of $M$ to the product of irreducible factors. We take $\phi \in A(M)$. In order to investigate $F(\phi, M)$ it is sufficient to consider the case where $M_{1}=\cdots=M_{k}$ and

$$
\phi\left(x_{1}, \ldots, x_{k}\right)=\left(\phi_{k}\left(x_{k}\right), \phi_{1}\left(x_{1}\right), \ldots, \phi_{k-1}\left(x_{k-1}\right)\right) \quad\left(x_{i} \in M_{i}\right),
$$

where $\phi_{i}: M_{i} \rightarrow M_{i+1}(1 \leq i \leq k-1)$ and $\phi_{k}: M_{k} \rightarrow M_{1}$ are holomorphically isometric maps. In this case we have

$$
\begin{aligned}
& F(\phi, M) \\
& =\left\{\left(x_{1}, \phi_{1}\left(x_{1}\right), \phi_{2} \phi_{1}\left(x_{1}\right), \ldots, \phi_{k-1} \cdots \phi_{1}\left(x_{1}\right)\right) \mid x_{1} \in F\left(\phi_{k} \cdots \phi_{1}, M_{1}\right)\right\} .
\end{aligned}
$$


Hence $F(\phi, M)$ is discrete if and only if $F\left(\phi_{k} \cdots \phi_{1}, M_{1}\right)$ is discrete. In the case $F(\phi, M)$ is an antipodal set of $M$ and

$$
\# F(\phi, M)=\# F\left(\phi_{k} \cdots \phi_{1}, M_{1}\right) \text {. }
$$

\section{THE INTERSECTION OF TWO REAL FORMS}

4.1. Characteristic elements associated with a root system. As we mentioned in Remark 3.4 the complex structure $J$ of a Hermitian symmetric space of compact type satisfies $\langle\alpha, J\rangle=0, \pm 1$ for any root $\alpha$. Based on the fact we define a characteristic element of a root system as follows.

Let $R$ be a root system of a finite dimensional vector space $\mathfrak{a}$ with an inner product $\langle$,$\rangle . Then J \in \mathfrak{a}-\{0\}$ is a characteristic element of the first kind or simply a characteristic element associated with $R$ if $\langle\lambda, J\rangle=0, \pm 1$ for any $\lambda \in R$. We denote by $W(R)$ the Weyl group of $R$. If $J$ is a characteristic element associated with $R$, then so are $-J$ and $s J$ for any $s \in W(R)$. In the sequel we assume that $R$ is irreducible. For a characteristic element $J$ we can take a fundamental system $\Pi=\left\{\alpha_{1}, \ldots, \alpha_{r}\right\}$ of $R$ such that $\left\langle\alpha_{i}, J\right\rangle=0,1$ for any $\alpha_{i}$. Denote by $\delta=\sum m_{i} \alpha_{i}$ the highest root of $R$. When the type of $R$ is one of $E_{8}, F_{4}$ and $G_{2}$, there does not exist a characteristic element since $m_{i} \geq 2$ for any $i$. In order to describe $W(R) J$ in detail we give the definition of a two-point homogeneous space, which appears in the following proposition. For a group $A$ which isometrically acts on a metric space $(X, d)$ if for any two pairs $x, y \in X$ and $x^{\prime}, y^{\prime} \in X$ satisfying $d(x, y)=d\left(x^{\prime}, y^{\prime}\right)$ there exists $a \in A$ such that $a x=x^{\prime}$, $a y=y^{\prime}$, then we call $X$ a two-point homogeneous space by the action of $A$. We prove the following proposition using the classification of an irreducible root system.

Proposition 4.1. The orbit $W(R) J$ of a characteristic element $J$ associated with an irreducible root system $R$ is a two-point homogeneous space by the action of $W(R)$.

Proof. Define a set $\left\{d_{1}, \ldots, d_{t}\right\}\left(0<d_{1}<\cdots<d_{t}\right)$ by

$$
\left\{d_{1}, \ldots, d_{t}\right\}=\{\|s J-J\| \mid s \in W(R)\}-\{0\} .
$$

The condition for $W(R) J$ to be two-point homogeneous is equivalent to the condition that the isotropy subgroup $\{s \in W(R) \mid s J=J\}$ acts transitively on each $\left\{s J \mid\|s J-J\|=d_{i}, s \in W(R)\right\}$. Denote by $C h(R)$ the set of all characteristic elements associated with $R$. We examine the condition above for each characteristic element $J$ associated with each $R=A_{r}, B_{r}, C_{r}, B C_{r}, D_{r}, E_{6}$ and $E_{7}$ in Examples 4.2, 4.3, 4.4, 4.5, 
4.6, 4.8, 4.10. If $R$ is the root system of $\mathfrak{g}$ in Section 2 , according to Theorem 2.1 the orbit $W(R) J$ is a great antipodal set of the Hermitian symmetric space of compact type associated with $J$, so we also calculate its cardinality $\#(W(R) J)$. We follow the same notations of the set of positive roots in [2]. Denote by $\left\{e_{1}, \ldots, e_{r}\right\}$ the standard orthonormal basis of $\mathbb{R}^{r}$.

Example 4.2. In the case where $R=B_{r}=\left\{ \pm e_{i} \pm e_{j}, \pm e_{i}\right\}$, set $J=e_{1}$. Then we have

$$
C h(R)=W(R) J=\left\{ \pm e_{1}, \ldots, \pm e_{r}\right\} .
$$

Thus $\#(W(R) J)=2 r, t=2, d_{1}=\sqrt{2}$ and $d_{2}=2$. We can verify that $W(R) J$ is two-point homogeneous.

Example 4.3. In the case where $R=C_{r}=\left\{ \pm e_{i} \pm e_{j}, \pm 2 e_{i}\right\}$, set

$$
J=\frac{1}{2}\left(e_{1}+e_{2}+\cdots+e_{r}\right)
$$

Then we have

$$
C h(R)=W(R) J=\left\{\frac{1}{2} \sum_{i=1}^{r} \epsilon_{i} e_{i} \mid \epsilon_{i}= \pm 1\right\} .
$$

Thus $\#(W(R) J)=2^{r}, t=r$ and $d_{i}=\sqrt{i} \quad(1 \leq i \leq r)$. We can verify that $W(R) J$ is two-point homogeneous.

Example 4.4. In the case where $R=B C_{r}=\left\{ \pm e_{i} \pm e_{j}, \pm e_{i}, \pm 2 e_{i}\right\}$, Examples 4.2 and 4.3 imply that there does not exist a characteristic element.

Example 4.5. In the case where $R=D_{r}=\left\{ \pm e_{i} \pm e_{j}\right\}$, we define characteristic elements $J_{1}, J_{2}$ and $J_{3}$ by

$$
J_{1}=e_{1}, \quad J_{2}=\frac{1}{2}\left(\sum_{j=1}^{r-1} e_{j}-e_{r}\right), \quad J_{3}=\frac{1}{2} \sum_{j=1}^{r} e_{j} .
$$

Then

$$
\begin{aligned}
& W(R) J_{1}=\left\{ \pm e_{1}, \ldots, \pm e_{r}\right\} \\
& W(R) J_{2}=\left\{\frac{1}{2} \sum_{j=1}^{r} \epsilon_{j} e_{j} \mid \epsilon_{j}= \pm 1, \epsilon_{1} \cdots \epsilon_{r}=-1\right\} \\
& W(R) J_{3}=\left\{\frac{1}{2} \sum_{j=1}^{r} \epsilon_{j} e_{j} \mid \epsilon_{j}= \pm 1, \epsilon_{1} \cdots \epsilon_{r}=1\right\}
\end{aligned}
$$


and $C h(R)=W(R) J_{1} \cup W(R) J_{2} \cup W(R) J_{3}$. Thus \# $\left(W(R) J_{1}\right)=2 r$, $\#\left(W(R) J_{2}\right)=\#\left(W(R) J_{3}\right)=2^{r-1}$. We can verify that $W(R) J_{i}$ is two-point homogeneous.

Example 4.6. In the case where $R=A_{r}=\left\{ \pm\left(e_{i}-e_{j}\right)\right\}$, we define characteristic elements $J_{1}, \ldots, J_{r}$ by

$$
J_{i}=\left(e_{1}+\cdots+e_{i}\right)-\frac{i}{r+1} \sum_{j=1}^{r+1} e_{j} .
$$

Then

$$
W(R) J_{i}=\left\{\sum_{j \in A} e_{j}-\frac{i}{r+1} \sum_{j=1}^{r+1} e_{j} \mid A \in P_{i}(r+1)\right\},
$$

where we put $P_{i}(r+1)=\{A \subset\{1,2, \ldots, r+1\} \mid \# A=i\}$. Thus $C h(R)=W(R) J_{1} \cup \cdots \cup W(R) J_{r}$ and $\#\left(W(R) J_{i}\right)=\left(\begin{array}{c}r+1 \\ i\end{array}\right)$. We can verify that $W(R) J_{i}$ is two-point homogeneous.

Remark 4.7. In the case of $A_{2}$, the orbit $W(R) J_{1}$ consists of three vertices of an equilateral triangle whose center of mass is the origin. Since $W(R) J_{2}=-W(R) J_{1}$, the above statement holds for $W(R) J_{2}$. These will be used later.

Example 4.8. In the case where $R=E_{6}$, we define characteristic elements $J_{1}$ and $J_{2}$ by

$$
\begin{aligned}
& J_{1}=\frac{2}{3}\left(e_{8}-e_{7}-e_{6}\right)=\frac{1}{3}\left(4 \alpha_{1}+3 \alpha_{2}+5 \alpha_{3}+6 \alpha_{4}+4 \alpha_{5}+2 \alpha_{6}\right), \\
& J_{2}=\frac{1}{3}\left(e_{8}-e_{7}-e_{6}\right)+e_{5}=\frac{1}{3}\left(2 \alpha_{1}+3 \alpha_{2}+4 \alpha_{3}+6 \alpha_{4}+5 \alpha_{5}+4 \alpha_{6}\right) .
\end{aligned}
$$

Then

$$
C h(R)=W(R) J_{1} \cup W(R) J_{2}, \quad W(R)\left(-J_{2}\right)=W(R) J_{1} .
$$

Lemma 2.2 implies that

$$
\#\left(W(R) J_{i}\right)=\frac{\#\left(W\left(\mathfrak{e}_{6}\right)\right)}{\#(W(\mathfrak{s o}(10)+\mathbb{R}))}=\frac{2^{7} \cdot 3^{4} \cdot 5}{2^{4} \cdot 5 !}=3^{3} .
$$

We can verify that $t=2, d_{1}=2, d_{2}=4$ and $W(R) J_{i}$ is two-point homogeneous.

Remark 4.9. There exist exactly five subsets $\Delta_{i}(1 \leq i \leq 5)$ of $W(R) J_{1}$ such that each $\Delta_{i}$ contains $J_{1}$ and consists of three vertices of an equilateral triangle whose center of mass is equal to the origin. If we set $\mathfrak{a}^{(i)}=\operatorname{span}_{\mathbb{R}}\left(\Delta_{i}\right)(1 \leq i \leq 5)$, then

$$
\Delta_{i}=\mathfrak{a}^{(i)} \cap W(R) J_{1}
$$


These will be used later.

Example 4.10. In the case where $R=E_{7}$, we define a characteristic element $J$ by

$$
\left\langle\alpha_{7}, J\right\rangle=1, \quad\left\langle\alpha_{i}, J\right\rangle=0(i \neq 7) .
$$

Then $C h(R)=W(R) J$. Lemma 2.2 implies that

$$
\#(W(R) J)=\frac{\#\left(W\left(\mathfrak{e}_{7}\right)\right)}{\#\left(W\left(\mathfrak{e}_{6}+\mathbb{R}\right)\right)}=\frac{2^{10} \cdot 3^{4} \cdot 5 \cdot 7}{2^{7} \cdot 3^{4} \cdot 5}=2^{3} \cdot 7 .
$$

We can verify that $t=3, d_{1}=\sqrt{2}, d_{2}=2, d_{3}=\sqrt{6}$ and $W(R) J$ is two-point homogeneous.

Hence we complete the proof of Proposition 4.1.

Theorem 2.1 and Proposition 4.1 imply the following theorem.

Theorem 4.11. A great antipodal set of an irreducible Hermitian symmetric space of compact type is a two-point homogeneous space.

4.2. Symmetric triads. In this subsection we review some results on symmetric triads obtained in [6] and [7]. These results will be used in Section 4.3 and Section 5.

Let $\mathfrak{a}$ be a finite dimensional vector space over $\mathbb{R}$ with an inner product $\langle$,$\rangle . A triple (\tilde{\Sigma}, \Sigma, W)$ is a symmetric triad of $\mathfrak{a}$, if it satisfies the following six conditions:

(1) $\tilde{\Sigma}$ is an irreducible root system of $\mathfrak{a}$, and $\tilde{\Sigma}$ spans $\mathfrak{a}$.

(2) $\Sigma$ is a root system of $\mathfrak{a}$.

(3) $W$ is a nonempty subset of $\mathfrak{a}$, which is invariant under the multiplication by -1 , and $\tilde{\Sigma}=\Sigma \cup W$.

(4) $\Sigma \cap W$ is a nonempty subset. If we put $l=\max \{\|\alpha\| \mid \alpha \in$ $\Sigma \cap W\}$, then $\Sigma \cap W=\{\alpha \in \tilde{\Sigma} \mid\|\alpha\| \leq l\}$.

(5) For $\alpha \in W, \lambda \in \Sigma-W, 2 \frac{\langle\alpha, \lambda\rangle}{\|\alpha\|^{2}}$ is odd if and only if $s_{\alpha} \lambda \in$ $W-\Sigma$, where we set $s_{\alpha} \lambda=\lambda-2 \frac{\langle\alpha, \lambda\rangle}{\|\alpha\|^{2}} \alpha$.

(6) For $\alpha \in W, \lambda \in W-\Sigma, 2 \frac{\langle\alpha, \lambda\rangle}{\|\alpha\|^{2}}$ is odd if and only if $s_{\alpha} \lambda \in$ $\Sigma-W$.

If $(\tilde{\Sigma}, \Sigma, W)$ is a symmetric triad of $\mathfrak{a}$, then $\Sigma$ spans $\mathfrak{a}$. In fact, using (4) we have

$\mathfrak{a} \supset \operatorname{span}(\Sigma) \supset \operatorname{span}(\Sigma \cap W) \supset \operatorname{span}\{$ the shortest roots in $\tilde{\Sigma}\}=\mathfrak{a}$.

For a symmetric triad $(\tilde{\Sigma}, \Sigma, W)$ of $\mathfrak{a}$, take a fundamental system $\tilde{\Pi}$ of $\tilde{\Sigma}$. Denote by $\tilde{\Sigma}^{+}$the set of positive roots in $\tilde{\Sigma}$ with respect to $\tilde{\Pi}$. If 
we put $\Sigma^{+}=\Sigma \cap \tilde{\Sigma}^{+}$and $W^{+}=W \cap \tilde{\Sigma}^{+}$, then $\Sigma=\Sigma^{+} \cup\left(-\Sigma^{+}\right)$and $W=W^{+} \cup\left(-W^{+}\right)$. We define a nonempty subset $\mathfrak{a}_{r}$ in $\mathfrak{a}$ by

$$
\mathfrak{a}_{r}=\bigcap_{\substack{\lambda \in \Sigma \\ \alpha \in W}}\left\{H \in \mathfrak{a} \mid\langle\lambda, H\rangle \notin \pi \mathbb{Z},\langle\alpha, H\rangle \notin \frac{\pi}{2}+\pi \mathbb{Z}\right\} .
$$

Then $\mathfrak{a}_{r}$ is an open dense subset of $\mathfrak{a}$. A point in $\mathfrak{a}_{r}$ is called a regular point.

Let $G$ be a connected compact simple Lie group and $\left(G, F_{1}, F_{2}\right)$ a compact symmetric triad: There exist two involutions $\theta_{1}$ and $\theta_{2}$ on $G$ such that the closed subgroup $F_{i}$ of $G$ lies between $F\left(\theta_{i}, G\right)$ and its identity component $F\left(\theta_{i}, G\right)_{0}$. We denote by $\mathfrak{g}, \mathfrak{f}_{1}$ and $\mathfrak{f}_{2}$ the Lie algebras of $G, F_{1}$ and $F_{2}$ respectively. We assume that $\theta_{1} \theta_{2}=\theta_{2} \theta_{1}$ and that $\theta_{1}$ cannot be transformed to $\theta_{2}$ by an inner automorphism of $G$. We denote the differential of $\theta_{i}$ by the same symbol $\theta_{i}$. We have two canonical decompositions of $\mathfrak{g}$ :

$$
\mathfrak{g}=\mathfrak{f}_{1} \oplus \mathfrak{p}_{1}=\mathfrak{f}_{2} \oplus \mathfrak{p}_{2},
$$

where $\mathfrak{p}_{i}=F\left(-\theta_{i}, \mathfrak{g}\right)$. Since $\theta_{1} \theta_{2}=\theta_{2} \theta_{1}$, we have

$$
\mathfrak{g}=\left(\mathfrak{f}_{1} \cap \mathfrak{f}_{2}\right) \oplus\left(\mathfrak{p}_{1} \cap \mathfrak{p}_{2}\right) \oplus\left(\mathfrak{f}_{1} \cap \mathfrak{p}_{2}\right) \oplus\left(\mathfrak{f}_{2} \cap \mathfrak{p}_{1}\right) .
$$

Take a maximal abelian subspace $\mathfrak{a}$ of $\mathfrak{p}_{1} \cap \mathfrak{p}_{2}$. The isometric action of $F_{1}$ on a Riemannian symmetric space $G / F_{2}$ of compact type is called a Hermann action. Since the action is a hyperpolar action whose section is the orbit of $A=\exp \mathfrak{a}$ through the origin, we have $G=F_{1} A F_{2}$. For each $\alpha \in \mathfrak{a}$ define a subspace $\mathfrak{g}(\mathfrak{a}, \alpha)$ of $\mathfrak{g}^{\mathbb{C}}$ by

$$
\mathfrak{g}(\mathfrak{a}, \alpha)=\left\{X \in \mathfrak{g}^{\mathbb{C}} \mid[H, X]=\sqrt{-1}\langle\alpha, H\rangle X(H \in \mathfrak{a})\right\}
$$

and set $\tilde{\Sigma}=\{\alpha \in \mathfrak{a}-\{0\} \mid \mathfrak{g}(\mathfrak{a}, \alpha) \neq\{0\}\}$. For $\epsilon= \pm 1$ define a subspace $\mathfrak{g}(\mathfrak{a}, \alpha, \epsilon)$ of $\mathfrak{g}(\mathfrak{a}, \alpha)$ by

$$
\mathfrak{g}(\mathfrak{a}, \alpha, \epsilon)=\left\{X \in \mathfrak{g}(\mathfrak{a}, \alpha) \mid \theta_{1} \theta_{2} X=\epsilon X\right\} .
$$

Since $\mathfrak{g}(\mathfrak{a}, \alpha)$ is $\theta_{1} \theta_{2}$-invariant, we have

$$
\mathfrak{g}(\mathfrak{a}, \alpha)=\mathfrak{g}(\mathfrak{a}, \alpha, 1) \oplus \mathfrak{g}(\mathfrak{a}, \alpha,-1) .
$$

Set $\Sigma=\{\alpha \in \tilde{\Sigma} \mid \mathfrak{g}(\mathfrak{a}, \alpha, 1) \neq\{0\}\}$ and $W=\{\alpha \in \tilde{\Sigma} \mid \mathfrak{g}(\mathfrak{a}, \alpha,-1) \neq$ $\{0\}\}$. Then the triple $(\tilde{\Sigma}, \Sigma, W)$ is a symmetric triad of $\mathfrak{a}$. Define closed subgroups $G_{12}$ and $F_{12}$ by $G_{12}=F\left(\theta_{1} \theta_{2}, G\right)$ and $F_{12}=\{g \in$ $\left.G_{12} \mid \theta_{1}(g)=g\right\}$. Then the Lie algebras of $G_{12}$ and $F_{12}$ are given by

$$
\mathfrak{g}_{12}=\left(\mathfrak{f}_{1} \cap \mathfrak{f}_{2}\right) \oplus\left(\mathfrak{p}_{1} \cap \mathfrak{p}_{2}\right), \quad \mathfrak{f}_{12}=\mathfrak{f}_{1} \cap \mathfrak{f}_{2},
$$


respectively. The restricted root system of the compact symmetric pair $\left(G_{12}, F_{12}\right)$ with respect to $\mathfrak{a}$ coincides with $\Sigma$. For $\lambda \in \Sigma$, we define subspaces $\mathfrak{p}_{\lambda}$ in $\mathfrak{p}_{1} \cap \mathfrak{p}_{2}$ and $\mathfrak{f}_{\lambda}$ in $\mathfrak{f}_{1} \cap \mathfrak{f}_{2}$ as follows:

$$
\begin{aligned}
\mathfrak{p}_{\lambda} & =\left\{X \in \mathfrak{p}_{1} \cap \mathfrak{p}_{2} \mid[H,[H, X]]=-\langle\lambda, H\rangle^{2} X(H \in \mathfrak{a})\right\}, \\
\mathfrak{f}_{\lambda} & =\left\{X \in \mathfrak{f}_{1} \cap \mathfrak{f}_{2} \mid[H,[H, X]]=-\langle\lambda, H\rangle^{2} X(H \in \mathfrak{a})\right\} .
\end{aligned}
$$

Take a maximal abelian subalgebra $\mathfrak{t}$ in $\mathfrak{g}_{12}$ containing $\mathfrak{a}$. Denote by $\tilde{R}$ the root system of $\mathfrak{g}_{12}$ with respect to $\mathfrak{t}$. Let $\mathfrak{t} \rightarrow \mathfrak{a} ; H \mapsto \bar{H}$ be the orthogonal projection and set $\tilde{R}_{0}=\{\alpha \in \tilde{R} \mid \bar{\alpha}=0\}$. Define a subalgebra $\mathfrak{f}_{0}$ in $\mathfrak{f}_{1} \cap \mathfrak{f}_{2}$ by

$$
\mathfrak{f}_{0}=\left\{X \in \mathfrak{f}_{1} \cap \mathfrak{f}_{2} \mid[\mathfrak{a}, X]=\{0\}\right\} .
$$

Take a compatible ordering of $\mathfrak{t}$. Then we have the following lemma.

Lemma 4.12. (1) We have orthogonal direct sum decompositions:

$$
\mathfrak{f}_{1} \cap \mathfrak{f}_{2}=\mathfrak{f}_{0} \oplus \sum_{\lambda \in \Sigma^{+}} \mathfrak{f}_{\lambda}, \quad \mathfrak{p}_{1} \cap \mathfrak{p}_{2}=\mathfrak{a} \oplus \sum_{\lambda \in \Sigma^{+}} \mathfrak{p}_{\lambda}
$$

(2) For each $\alpha \in \tilde{R}^{+}-\tilde{R}_{0}$ there exist $S_{\alpha} \in \mathfrak{f}_{1} \cap \mathfrak{f}_{2}$ and $T_{\alpha} \in \mathfrak{p}_{1} \cap \mathfrak{p}_{2}$ such that

$$
\left\{S_{\alpha} \mid \alpha \in \tilde{R}^{+}, \bar{\alpha}=\lambda\right\}, \quad\left\{T_{\alpha} \mid \alpha \in \tilde{R}^{+}, \bar{\alpha}=\lambda\right\}
$$

are respectively orthonormal bases of $\mathfrak{f}_{\lambda}$ and $\mathfrak{p}_{\lambda}$, and for $H \in \mathfrak{a}$

$$
\begin{aligned}
& {\left[H, S_{\alpha}\right]=\langle\alpha, H\rangle T_{\alpha}, \quad\left[H, T_{\alpha}\right]=-\langle\alpha, H\rangle S_{\alpha}, \quad\left[S_{\alpha}, T_{\alpha}\right]=\bar{\alpha},} \\
& \operatorname{Ad}(\exp H) S_{\alpha}=\cos \langle\alpha, H\rangle S_{\alpha}+\sin \langle\alpha, H\rangle T_{\alpha}, \\
& \operatorname{Ad}(\exp H) T_{\alpha}=-\sin \langle\alpha, H\rangle S_{\alpha}+\cos \langle\alpha, H\rangle T_{\alpha} .
\end{aligned}
$$

Define subspaces of $\mathfrak{f}_{1} \cap \mathfrak{p}_{2}$ and $\mathfrak{p}_{1} \cap \mathfrak{f}_{2}$ by

$$
\begin{aligned}
V\left(\mathfrak{f}_{1} \cap \mathfrak{p}_{2}\right) & =\left\{X \in \mathfrak{f}_{1} \cap \mathfrak{p}_{2} \mid[\mathfrak{a}, X]=\{0\}\right\}, \\
V\left(\mathfrak{p}_{1} \cap \mathfrak{f}_{2}\right) & =\left\{X \in \mathfrak{p}_{1} \cap \mathfrak{f}_{2} \mid[\mathfrak{a}, X]=\{0\}\right\}, \\
V^{\perp}\left(\mathfrak{f}_{1} \cap \mathfrak{p}_{2}\right) & =\left\{X \in \mathfrak{f}_{1} \cap \mathfrak{p}_{2} \mid X \perp V\left(\mathfrak{f}_{1} \cap \mathfrak{p}_{2}\right)\right\}, \\
V^{\perp}\left(\mathfrak{p}_{1} \cap \mathfrak{f}_{2}\right) & =\left\{X \in \mathfrak{p}_{1} \cap \mathfrak{f}_{2} \mid X \perp V\left(\mathfrak{p}_{1} \cap \mathfrak{f}_{2}\right)\right\} .
\end{aligned}
$$

For $\alpha \in W$ define subspaces $V_{\alpha}^{\perp}\left(\mathfrak{f}_{1} \cap \mathfrak{p}_{2}\right)$ in $V^{\perp}\left(\mathfrak{f}_{1} \cap \mathfrak{p}_{2}\right)$ and $V_{\alpha}^{\perp}\left(\mathfrak{p}_{1} \cap \mathfrak{f}_{2}\right)$ in $V^{\perp}\left(\mathfrak{p}_{1} \cap \mathfrak{f}_{2}\right)$ by

$$
\begin{aligned}
& V_{\alpha}^{\perp}\left(\mathfrak{f}_{1} \cap \mathfrak{p}_{2}\right)=\left\{X \in V^{\perp}\left(\mathfrak{f}_{1} \cap \mathfrak{p}_{2}\right) \mid[H,[H, X]]=-\langle\alpha, H\rangle^{2} X(H \in \mathfrak{a})\right\}, \\
& V_{\alpha}^{\perp}\left(\mathfrak{p}_{1} \cap \mathfrak{f}_{2}\right)=\left\{X \in V^{\perp}\left(\mathfrak{p}_{1} \cap \mathfrak{f}_{2}\right) \mid[H,[H, X]]=-\langle\alpha, H\rangle^{2} X(H \in \mathfrak{a})\right\} .
\end{aligned}
$$

Then we have the orthogonal direct sum decompositions:

$$
V^{\perp}\left(\mathfrak{f}_{1} \cap \mathfrak{p}_{2}\right)=\sum_{\alpha \in W^{+}} V_{\alpha}^{\perp}\left(\mathfrak{f}_{1} \cap \mathfrak{p}_{2}\right), \quad V^{\perp}\left(\mathfrak{p}_{1} \cap \mathfrak{f}_{2}\right)=\sum_{\alpha \in W^{+}} V_{\alpha}^{\perp}\left(\mathfrak{p}_{1} \cap \mathfrak{f}_{2}\right) .
$$


For $\lambda \in \Sigma$ and $\alpha \in W$, set

$$
m(\lambda)=\operatorname{dim}_{\mathbb{C}} \mathfrak{g}(\mathfrak{a}, \lambda, 1), \quad n(\alpha)=\operatorname{dim}_{\mathbb{C}} \mathfrak{g}(\mathfrak{a}, \alpha,-1) .
$$

Lemma 4.13. (1) For any $\alpha \in W^{+}$,

$$
\begin{aligned}
& {\left[\mathfrak{a}, V_{\alpha}^{\perp}\left(\mathfrak{f}_{1} \cap \mathfrak{p}_{2}\right)\right]=V_{\alpha}^{\perp}\left(\mathfrak{p}_{1} \cap \mathfrak{f}_{2}\right),} \\
& {\left[\mathfrak{a}, V_{\alpha}^{\perp}\left(\mathfrak{p}_{1} \cap \mathfrak{f}_{2}\right)\right]=V_{\alpha}^{\perp}\left(\mathfrak{f}_{1} \cap \mathfrak{p}_{2}\right) .}
\end{aligned}
$$

(2) There exist orthonormal bases $\left\{X_{\alpha, i}\right\}_{1 \leq i \leq n(\alpha)}$ and $\left\{Y_{\alpha, i}\right\}_{1 \leq i \leq n(\alpha)}$ of $V_{\alpha}^{\perp}\left(\mathfrak{f}_{1} \cap \mathfrak{p}_{2}\right)$ and $V_{\alpha}^{\perp}\left(\mathfrak{p}_{1} \cap \mathfrak{f}_{2}\right)$ respectively such that, for any $H \in \mathfrak{a}$,

$$
\begin{aligned}
& {\left[H, X_{\alpha, i}\right]=\langle\alpha, H\rangle Y_{\alpha, i}, \quad\left[H, Y_{\alpha, i}\right]=-\langle\alpha, H\rangle X_{\alpha, i},} \\
& {\left[X_{\alpha, i}, Y_{\alpha, i}\right]=\alpha,} \\
& \operatorname{Ad}(\exp H) X_{\alpha, i}=\cos \langle\alpha, H\rangle X_{\alpha, i}+\sin \langle\alpha, H\rangle Y_{\alpha, i}, \\
& \operatorname{Ad}(\exp H) Y_{\alpha, i}=-\sin \langle\alpha, H\rangle X_{\alpha, i}+\cos \langle\alpha, H\rangle Y_{\alpha, i} .
\end{aligned}
$$

Lemmas 4.12 and 4.13 imply that

$$
W(\tilde{\Sigma}) \subset\left\{\left.\operatorname{Ad}(g)\right|_{\mathfrak{a}} \mid g \in G, \operatorname{Ad}(g) \mathfrak{a}=\mathfrak{a}\right\},
$$

where $W(\tilde{\Sigma})$ denotes the Weyl group of $\tilde{\Sigma}$. See Corollary 4.17 and Lemma 4.4 in $[6]$ for the detail.

Take a maximal abelian subspace $\mathfrak{a}_{i}$ of $\mathfrak{p}_{i}$ containing $\mathfrak{a}$. The maximality of $\mathfrak{a}$ implies that $\mathfrak{a}=\mathfrak{a}_{1} \cap \mathfrak{a}_{2}$. Denote by $R_{i}$ the restricted root system of $\left(G, F_{i}\right)$ with respect to $\mathfrak{a}_{i}$. If $\mathfrak{a}=\mathfrak{a}_{1}$, then $\tilde{\Sigma}=R_{1}$. We list some $\left(G, F_{1}, F_{2}\right)$ 's, their symmetric triad $(\tilde{\Sigma}, \Sigma, W)$ 's, the restricted root system $\left(R_{i}\right)$ 's, and the structure of $\mathfrak{f}_{0}$ 's as a compact Lie algebra, which we will need in Subsection 4.3 and Section 5.

\begin{tabular}{|l|l|l|}
\hline & $\left(G, F_{1}, F_{2}\right)$ & $(\tilde{\Sigma}, \Sigma, W)$ \\
\hline$(1)$ & $(S U(2 n), S(U(n) \times U(n)), S O(2 n))$ & $\left(\right.$ I'- $\left.C_{n}\right)$ \\
\hline$(2)$ & $(S p(2 m), S p(m) \times S p(m), U(2 m))$ & $\left(\right.$ III- $\left.C_{m}\right)$ \\
\hline$(3)$ & $(S O(4 m), U(2 m), S O(2 m) \times S O(2 m))$ & $\left(\right.$ I- $\left.C_{m}\right)$ \\
\hline$(4)$ & $\left(E_{7}, S^{1} \cdot E_{6}, S U(8)\right)$ & $\left(\right.$ I- $\left.C_{3}\right)$ \\
\hline$(5)$ & $(S O(r+s+t), S O(r) \times S O(s+t)$, & $\left(\right.$ I- $\left.B_{r}\right)$ \\
& $S O(r+s) \times S O(t))(s>0, r<t)$ & \\
\hline$(6)$ & $\left(E_{6}, F_{4}, S p(4)\right)$ & $\left(\right.$ III- $\left.A_{2}\right)$ \\
\hline$(7)$ & $(S U(2(m+q)), S p(m+q), S O(2(m+q)))$ & $\left(\right.$ III- $\left.A_{m+q-1}\right)$ \\
\hline$(8)$ & $(S U(4 m), S p(2 m), S(U(2 m) \times U(2 m)))$ & $\left(\right.$ III- $\left.C_{m}\right)$ \\
\hline
\end{tabular}




\begin{tabular}{|l|l|l|l|}
\hline & $R_{1}$ & $R_{2}$ & $\mathfrak{f}_{0}$ \\
\hline$(1)$ & $C_{n}$ & $A_{2 n-1}$ & $\{0\}$ \\
\hline$(2)$ & $C_{m}$ & $C_{2 m}$ & $\mathbb{R}^{m}$ \\
\hline$(3)$ & $C_{m}$ & $D_{2 m}$ & $\mathbb{R}^{m}$ \\
\hline$(4)$ & $C_{3}$ & $E_{7}$ & $\mathfrak{s u}(2)^{4}$ \\
\hline$(5)$ & $B_{r}$ & $\begin{array}{l}B_{\min \{r+s, t\}}(\text { if } r+s \neq t) \\
D_{t}(\text { if } r+s=t)\end{array}$ & $\mathfrak{s o}(s) \oplus \mathfrak{s o}(t-r)$ \\
\hline$(6)$ & $A_{2}$ & $E_{6}$ & $\mathfrak{s p}(1)^{4}$ \\
\hline$(7)$ & $A_{m+q-1}$ & $A_{2(m+q)-1}$ & $\mathbb{R}^{m+q}$ \\
\hline$(8)$ & $A_{2 m-1}$ & $C_{2 m}$ & $\mathfrak{s p}(1)^{m}$ \\
\hline
\end{tabular}

We explain the notations in the table above. In column of $(\tilde{\Sigma}, \Sigma, W)^{\prime}$ s we used the following notations.

\begin{tabular}{|l|l|l|l|}
\hline & $\tilde{\Sigma}$ & $\Sigma$ & $W$ \\
\hline$(\mathrm{III}-\tilde{\Sigma})$ & $\tilde{\Sigma}$ & $\tilde{\Sigma}$ & $\tilde{\Sigma}$ \\
\hline$\left(\mathrm{I}-C_{n}\right)$ & $C_{n}$ & $D_{n}$ & $C_{n}$ \\
\hline$\left(\mathrm{I}-C_{m}\right)$ & $C_{m}$ & $C_{m}$ & $D_{m}$ \\
\hline$\left(\mathrm{I}-B_{r}\right)$ & $B_{r}$ & $B_{r}$ & $\left\{ \pm e_{i}\right\}$ \\
\hline
\end{tabular}

In the table above, $\mathfrak{f}_{0}=\mathbb{R}^{m}$ means that $\mathfrak{f}_{0}$ is an abelian Lie algebra of dimension $m$. We used table 1 in Tamaru [14] and a table of Section 4 in Matsuki [10] to determine the structure of $\mathfrak{f}_{0}$ when $G$ is of exceptional type. Note that $\mathfrak{s o}(s)$ is abelian if and only if $s \leq 2$.

4.3. The intersection of two real forms. Let $M=\operatorname{Ad}(G) J \subset \mathfrak{g}$ be an irreducible Hermitian symmetric space of compact type. In this subsection we study a necessary and sufficient condition that the intersection of two real forms of $M$ is discrete, and describe the intersection when it is discrete. Any two real forms of $M$ always intersect. Let $L_{1}=F\left(\tau_{1}, M\right)$ and $L_{2}=F\left(\tau_{2}, M\right)$ be two real forms of $M$, where $\tau_{i}$ is an involutive anti-holomorphic isometry of $M$. Define an involution $\theta_{i}$ of $G$ by $\theta_{i}(g)=\tau_{i} g \tau_{i}^{-1}$. If we set $F_{i}=F\left(\theta_{i}, G\right)$, then $\left(G, F_{1}, F_{2}\right)$ is a compact symmetric triad. In order to study $L_{1} \cap \operatorname{Ad}(a) L_{2}$ for $a \in G$, we may assume that $\tau_{1} \tau_{2}=\tau_{2} \tau_{1}$ by the classification of real forms in irreducible Hermitian symmetric spaces of compact type. We use the same notation in Subsection 4.2. Take a maximal abelian subspace $\mathfrak{a}$ of $\mathfrak{p}_{1} \cap \mathfrak{p}_{2}$ which contains $J$. We may assume that $a$ is in exp $\mathfrak{a}$ since $G=F_{1}(\exp \mathfrak{a}) F_{2}$. By Theorem 4.3 in [16], we have

$$
\begin{aligned}
& L_{1}=M \cap \mathfrak{p}_{1}, \quad \operatorname{Ad}(a) L_{2}=M \cap \operatorname{Ad}(a) \mathfrak{p}_{2}, \\
& L_{1} \cap \operatorname{Ad}(a) L_{2}=M \cap\left(\mathfrak{p}_{1} \cap \operatorname{Ad}(a) \mathfrak{p}_{2}\right) .
\end{aligned}
$$


If $L_{1}$ is congruent to $L_{2}$, which means that there exists $g \in G$ such that $L_{2}=\operatorname{Ad}(g) L_{1}$, then we may assume that $L_{1}=L_{2}$. We divide into the following two cases:

(1) $L_{1}=L_{2}$.

(2) $L_{1}$ is not congruent to $L_{2}$.

First we assume that $L_{1}=L_{2}$. Set $\tau=\tau_{1}=\tau_{2}, L=L_{1}=L_{2}$ and so on. Denote by $R$ the restricted root system of $(G, F)$ with respect to $\mathfrak{a}$. Then we have root space decompositions of $\mathfrak{f}$ and $\mathfrak{p}$ :

$$
\mathfrak{f}=\mathfrak{f}_{0} \oplus \sum_{\lambda \in R_{+}} \mathfrak{f}_{\lambda}, \quad \mathfrak{p}=\mathfrak{a} \oplus \sum_{\lambda \in R_{+}} \mathfrak{p}_{\lambda}
$$

where $R_{+}$is the set of positive roots in $R$ with respect to a lexicographic ordering. The complex structure $J$ is a characteristic element associated with $R$. Since

$$
\mathfrak{m}=[J, \mathfrak{g}]=\sum_{\substack{\lambda \in R_{+} \\\langle\lambda, J\rangle \neq 0}}\left(\mathfrak{f}_{\lambda} \oplus \mathfrak{p}_{\lambda}\right),
$$

we have

$$
\operatorname{dim} M=2 \sum_{\substack{\lambda \in R_{+} \\\langle\lambda, J\rangle \neq 0}} m_{R}(\lambda),
$$

where we denote by $m_{R}(\lambda)$ the multiplicity of $\lambda$. If we set $a=\exp H$ for $H \in \mathfrak{a}$, then

$$
\mathfrak{p} \cap \operatorname{Ad}(a) \mathfrak{p}=\mathfrak{a} \oplus \sum_{\substack{\lambda \in R_{+} \\\langle\lambda, H\rangle \in \pi \mathbb{Z}}} \mathfrak{p}_{\lambda} .
$$

Theorem 4.14. The intersection $L \cap \operatorname{Ad}(a) L$ is discrete if and only if $\langle\lambda, H\rangle \notin \pi \mathbb{Z}$ for any $\lambda \in R$. In this case,

$$
L \cap \operatorname{Ad}(a) L=M \cap \mathfrak{a}=W(R) J,
$$

where $M \cap \mathfrak{a}$ is a great antipodal set of $L$.

Proof. If $\langle\lambda, H\rangle \notin \pi \mathbb{Z}$ for any $\lambda \in R$, then we have (4.6), since $\mathfrak{p} \cap$ $\operatorname{Ad}(a) \mathfrak{p}=\mathfrak{a}$. Here the second equality follows from Proposition 2.2 in [5, Ch.VII].

If there exists $\lambda \in R$ such that $\langle\lambda, H\rangle \in \pi \mathbb{Z}$, then there exists $X \in$ $W(R) J$ such that $\langle\lambda, X\rangle \neq 0$. There exist unit vectors $S_{\lambda} \in \mathfrak{f}_{\lambda}$ and $T_{\lambda} \in \mathfrak{p}_{\lambda}$ such that for any $H^{\prime} \in \mathfrak{a}$

$$
\left[H^{\prime}, S_{\lambda}\right]=\left\langle\lambda, H^{\prime}\right\rangle T_{\lambda}, \quad\left[H^{\prime}, T_{\lambda}\right]=-\left\langle\lambda, H^{\prime}\right\rangle S_{\lambda}, \quad\left[S_{\lambda}, T_{\lambda}\right]=\lambda .
$$


Then

$$
\begin{aligned}
\operatorname{Ad}\left(\exp t S_{\lambda}\right) X & =X+\frac{\langle\lambda, X\rangle}{\|\lambda\|^{2}}(\cos (t\|\lambda\|)-1) \lambda-\frac{\langle\lambda, X\rangle}{\|\lambda\|} \sin (t\|\lambda\|) T_{\lambda} \\
& \in L_{1} \cap \operatorname{Ad}(a) L_{2} .
\end{aligned}
$$

Hence $L_{1} \cap \operatorname{Ad}(a) L_{2}$ is not discrete.

Example 4.15. If $(M, L)=(S p(r) / U(r), U(r) / O(r))$, then $R=C_{r}$ and $\#(W(R) J)=2^{r}$.

Proof. The assertion immediately follows from the table in Subsection 4.2 and Example 4.3.

Example 4.16. If $(M, L)=\left(G_{k}\left(\mathbb{C}^{n}\right), G_{k}\left(\mathbb{R}^{n}\right)\right)$, then $R=A_{n-1}$ and $\#(W(R) J)=\left(\begin{array}{l}n \\ k\end{array}\right)$.

Proof. Since $(G, F)=(S U(n), S O(n))$, we have $R=A_{n-1}$ and the multiplicity of any root in $R$ is equal to 1 . (4.5) implies that $J=J_{k}$ or $J=J_{n-k}$ in Example 4.6. Thus \# $(W(R) J)=\left(\begin{array}{l}n \\ k\end{array}\right)$ by Example 4.6.

Example 4.17. If $(M, L)=(S O(2 r) / U(r), S O(r))$, then $R=D_{r}$ and $\#(W(R) J)=2^{r-1}$.

Proof. Since $(G, F)=(S O(2 r), S(O(r) \times O(r)))$, we have $R=D_{r}$ and the multiplicity of any root in $R$ is equal to 1 . (4.5) implies that $J=J_{2}$ in Example 4.5. Thus \# $(W(R) J)=2^{r-1}$ by Example 4.5.

Next we assume that $L_{1}$ is not congruent to $L_{2}$. Denote by $(\tilde{\Sigma}, \Sigma, W)$ the symmetric triad associated with $\left(G, F_{1}, F_{2}\right)$. By Lemmas 4.12 and $4.13 J$ is a characteristic element associated with $\tilde{\Sigma}$. Lemmas 4.12 and 4.13 also imply that

$$
\mathfrak{p}_{1} \cap \operatorname{Ad}(a) \mathfrak{p}_{2}=\mathfrak{a} \oplus \sum_{\substack{\lambda \in \Sigma^{+} \\\langle\lambda, H\rangle \in \pi \mathbb{Z}}} \mathfrak{p}_{\lambda} \oplus \sum_{\substack{\alpha \in W^{+} \\\langle\alpha, H\rangle \in \frac{\pi}{2}+\pi \mathbb{Z}}} V_{\alpha}^{\perp}\left(\mathfrak{p}_{1} \cap \mathfrak{f}_{2}\right) .
$$

Denote by $R_{i}$ the restricted root system of $\left(G, F_{i}\right)$ with respect to $\mathfrak{a}_{i}$.

Theorem 4.18. The intersection $L_{1} \cap \operatorname{Ad}(a) L_{2}(a=\exp H)$ is discrete if and only if $H$ is a regular point of $(\tilde{\Sigma}, \Sigma, W)$.

Proof. If $H$ is a regular point of $(\tilde{\Sigma}, \Sigma, W)$, then $\mathfrak{p}_{1} \cap \operatorname{Ad}(a) \mathfrak{p}_{2}=\mathfrak{a}$. Thus

$$
L_{1} \cap \operatorname{Ad}(a) L_{2}=\left(M \cap \mathfrak{a}_{1}\right) \cap\left(M \cap \mathfrak{a}_{2}\right)=W\left(R_{1}\right) J \cap W\left(R_{2}\right) J .
$$

We assume that $H$ is not a regular point of $(\tilde{\Sigma}, \Sigma, W)$. Then (i) there exists $\lambda \in \Sigma$ such that $\langle\lambda, H\rangle \in \pi \mathbb{Z}$, or, (ii) there exists $\alpha \in W$ such that $\langle\alpha, H\rangle \in \frac{\pi}{2}+\pi \mathbb{Z}$. 
In the case of (i) we can prove that $L_{1} \cap \operatorname{Ad}(a) L_{2}$ is not discrete in a similar manner of the proof of Theorem 4.14. In the case of (ii) there exists $X \in W(\Sigma) J$ such that $\langle\alpha, X\rangle \neq 0$ since $W(\Sigma) \alpha$ spans a. Lemma 4.13 implies that

$$
\begin{aligned}
\operatorname{Ad}\left(\exp t X_{\alpha, i}\right) X & =X+\frac{\langle\alpha, X\rangle}{\|\alpha\|^{2}}(\cos (t\|\alpha\|)-1) \alpha-\frac{\langle\alpha, X\rangle}{\|\alpha\|} \sin (t\|\alpha\|) Y_{\alpha, i} \\
& \in L_{1} \cap \operatorname{Ad}(a) L_{2} .
\end{aligned}
$$

Hence $L_{1} \cap \operatorname{Ad}(a) L_{2}$ is not discrete.

Note that $H$ is a regular point of $(\tilde{\Sigma}, \Sigma, W)$ if and only if $F_{2}$-orbit through $a F_{1} \in G / F_{1}$ is a regular orbit.

In the sequel we assume that $L_{1} \cap \operatorname{Ad}(a) L_{2}$ is discrete. (4.4) and (4.7) imply

$$
W(\tilde{\Sigma}) J \subset M \cap \mathfrak{a}=L_{1} \cap \operatorname{Ad}(a) L_{2} \subset W\left(R_{i}\right) J \cap \mathfrak{a} .
$$

Based on the fact, we prove the following theorem.

Theorem 4.19. Assume that $L_{1} \cap \operatorname{Ad}(a) L_{2}$ is discrete. Then

$$
L_{1} \cap \operatorname{Ad}(a) L_{2}=W(\tilde{\Sigma}) J=W\left(R_{1}\right) J \cap \mathfrak{a}=W\left(R_{2}\right) J \cap \mathfrak{a} .
$$

Proof. It is sufficient to prove that $W\left(R_{i}\right) J \cap \mathfrak{a} \subset W(\tilde{\Sigma}) J$. The type of $\tilde{\Sigma}$ is $A, B$ or $C$ by the classification of $\left(M, L_{1}, L_{2}\right)$. If $\tilde{\Sigma}$ is of type $B$ or $C$, then $W(\tilde{\Sigma})=C h(\tilde{\Sigma})$ by Examples 4.2 and 4.3. Any $X \in W\left(R_{i}\right) J \cap \mathfrak{a}$ satisfies $(\operatorname{ad} X)^{3}=-\operatorname{ad} X$. Hence $X$ is in $C h(\tilde{\Sigma})$. If $\tilde{\Sigma}$ is of type $A$, then $\left(M, L_{1}, L_{2}\right)=\left(E_{6} / S^{1} \cdot \operatorname{Spin}(10), F_{4} / \operatorname{Spin}(9), G_{2}\left(\mathbb{H}^{4}\right) / \mathbb{Z}_{2}\right)$ or $\left(G_{2 q}\left(\mathbb{C}^{2(m+q)}\right), G_{q}\left(\mathbb{H}^{m+q}\right), G_{2 q}\left(\mathbb{R}^{2(m+q)}\right)\right)$. In these cases we will prove $W(\tilde{\Sigma}) J=W\left(R_{i}\right) J \cap \mathfrak{a}$ below.

\section{Example 4.20. If}

$$
\left(M, L_{1}, L_{2}\right)=\left(E_{6} / S^{1} \cdot \operatorname{Spin}(10), F_{4} / \operatorname{Spin}(9), G_{2}\left(\mathbb{H}^{4}\right) / \mathbb{Z}_{2}\right),
$$

then

$$
\mathfrak{a}_{1}=\mathfrak{a}, \quad \tilde{\Sigma}=R_{1}=A_{2}, \quad R_{2}=E_{6}
$$

and

$$
\begin{aligned}
& W(\tilde{\Sigma}) J=W\left(R_{1}\right) J=W\left(R_{2}\right) J \cap \mathfrak{a}, \\
& \#(W(\tilde{\Sigma}) J)=3, \quad \#\left(W\left(R_{2}\right) J\right)=3^{3} .
\end{aligned}
$$

Proof. Since $\left(G, F_{1}, F_{2}\right)=\left(E_{6}, F_{4}, S p(4)\right)$ we have $\tilde{\Sigma}=R_{1}=A_{2}$ and $R_{2}=E_{6}$ by $(6)$ of the table in Subsection 4.2. Hence $W(\tilde{\Sigma}) J=$ $W\left(R_{1}\right) J \cap \mathfrak{a}=W\left(R_{1}\right) J$. From Example 4.6 we have $\#(W(\tilde{\Sigma}) J)=3$. There exists $i(1 \leq i \leq 5)$ such that $\mathfrak{a}_{2}=\mathfrak{a}^{(i)}$ by Remarks 4.7 and 4.9 . 
Hence $\#\left(W\left(R_{2}\right) J \cap \mathfrak{a}\right)=3$ by Remark 4.9. From Example 4.8 we have $\#\left(W\left(R_{2}\right) J\right)=3^{3}$.

Example 4.21. If

$$
\left(M, L_{1}, L_{2}\right)=\left(G_{2 q}\left(\mathbb{C}^{2(m+q)}\right), G_{q}\left(\mathbb{H}^{m+q}\right), G_{2 q}\left(\mathbb{R}^{2(m+q)}\right)\right),
$$

then

$$
\mathfrak{a}_{1}=\mathfrak{a}, \quad \tilde{\Sigma}=R_{1}=A_{m+q-1}, \quad R_{2}=A_{2(m+q)-1}
$$

and

$$
\begin{aligned}
& W(\tilde{\Sigma}) J=W\left(R_{1}\right) J=W\left(R_{2}\right) J \cap \mathfrak{a}, \\
& \#(W(\tilde{\Sigma}) J)=\left(\begin{array}{c}
m+q \\
q
\end{array}\right), \quad \#\left(W\left(R_{2}\right) J\right)=\left(\begin{array}{c}
2 m+2 q \\
2 m
\end{array}\right) .
\end{aligned}
$$

Proof. We have $\mathfrak{a}_{1}=\mathfrak{a}, \tilde{\Sigma}=R_{1}=A_{m+q-1}, R_{2}=A_{2(m+q)-1}$ by $(7)$ of the table in Subsection 4.2. Hence $W(\tilde{\Sigma}) J=W\left(R_{1}\right) J$. Since $J$ is a characteristic element associated with $R_{1}$, there exists $J_{i}(1 \leq i \leq$ $m+q-1)$ in Example 4.6 such that $J=J_{i}$. Since the multiplicity of any root in $R_{1}$ is equal to $4,(4.5)$ implies that

$$
8 m q=\operatorname{dim} M=2 \cdot 4 \cdot i(m+q-i) .
$$

Hence $i=m$ or $i=q$. In any case we have

$$
\#(W(\tilde{\Sigma}) J)=\left(\begin{array}{c}
m+q \\
q
\end{array}\right)
$$

by Example 4.6. Since $J$ is also a characteristic element associated with $R_{2}$, there exists $J_{k}(1 \leq k \leq 2 m+2 q-1)$ in Example 4.6 such that $J=J_{k}$. Since the multiplicity of any root in $R_{2}$ is equal to 1 , (4.5) implies that

$$
8 m q=\operatorname{dim} M=2 \cdot k(2 m+2 q-k) .
$$

Hence $k=2 m$ or $k=2 q$. In any case we have

$$
\#\left(W\left(R_{2}\right) J\right)=\left(\begin{array}{c}
2 m+2 q \\
2 m
\end{array}\right)
$$

by Example 4.6. To show $W(\tilde{\Sigma}) J=W\left(R_{2}\right) J \cap \mathfrak{a}$, we identify $\mathfrak{a}_{2}$ and $\mathfrak{a}$ with the following subspaces.

$$
\begin{aligned}
& \mathfrak{a}_{2} \cong\left\{\begin{array}{l|l}
\sum_{i=1}^{p+q}\left(x_{i} e_{i}+y_{i} e_{i+p+q}\right) \in \mathbb{R}^{2(m+q)} & \sum_{i=1}^{p+q}\left(x_{i}+y_{i}\right)=0
\end{array}\right\}, \\
& \mathfrak{a} \cong\left\{\begin{array}{l|l}
\sum_{i=1}^{p+q} x_{i}\left(e_{i}+e_{i+p+q}\right) \in \mathbb{R}^{2(m+q)} & \sum_{i=1}^{p+q} x_{i}=0
\end{array}\right\} \text {. }
\end{aligned}
$$


Under the identification we have $R_{2}^{+} \cong\left\{e_{i}-e_{j} \mid 1 \leq i<j \leq 2 m+2 q\right\}$. Since $J=J_{2 m}$ of $J=J_{2 q}$, set $J=J_{2 m}$ for instance. In order to describe $W\left(R_{2}\right) J$ we use the following notations. Denote by $P_{a}(m+q)$ the set consisting of all subsets of cardinality $a$ in $\{1,2, \ldots, m+q\}$. For $A \in P_{a}(m+q)$ denote by $\bar{A}$ the complement of $A$ in $\{1, \ldots, m+q\}$ and set $A^{\prime}=\{x+m+q \mid x \in A\}$. For any $X \in W\left(R_{2}\right) J$ there exist $A \in P_{a}(m+q)$ and $B \in P_{b}(m+q)$ such that $a+b=2 m$ and that

$$
\begin{aligned}
X & =\sum_{j \in A} e_{j}+\sum_{k \in B^{\prime}} e_{k}-\frac{m}{m+q}\left(\sum_{j \in A} e_{j}+\sum_{j \in \bar{A}} e_{j}+\sum_{k \in \bar{B}} e_{k}+\sum_{k \in \bar{B}^{\prime}} e_{k}\right) \\
& =\frac{q}{m+q}\left(\sum_{j \in A} e_{j}+\sum_{k \in \bar{B}} e_{k}\right)-\frac{m}{m+q}\left(\sum_{j \in \bar{A}} e_{j}+\sum_{k \in \bar{B}^{\prime}} e_{k}\right) .
\end{aligned}
$$

Hence $X$ is in $\mathfrak{a}$ if and only if $a=b=m, A=B$. In this case

$$
X=\frac{q}{m+q} \sum_{j \in A}\left(e_{j}+e_{j+m+q}\right)-\frac{q}{m+q} \sum_{j \in \bar{A}}\left(e_{j}+e_{j+m+q}\right) .
$$

Hence we have

$$
\#\left(W\left(R_{2}\right) J \cap \mathfrak{a}\right)=\left(\begin{array}{c}
m+q \\
q
\end{array}\right) .
$$

Thus $W(\tilde{\Sigma}) J=W\left(R_{2}\right) J \cap \mathfrak{a}$. When $J=J_{2 q}$, we get the same conclusion.

This completes the proof of Theorem 4.19.

We give another example.

Example 4.22. If

$$
\left(M, L_{1}, L_{2}\right)=\left(G_{2 m}\left(\mathbb{C}^{4 m}\right), G_{m}\left(\mathbb{H}^{2 m}\right), U(2 m)\right),
$$

then $\tilde{\Sigma}=C_{m}, R_{1}=A_{2 m-1}, R_{2}=C_{2 m}$ and

$\#\left(L_{1} \cap \operatorname{Ad}(a) L_{2}\right)=2^{m}, \quad \#\left(W\left(R_{1}\right) J\right)=\left(\begin{array}{c}2 m \\ m\end{array}\right), \quad \#\left(W\left(R_{2}\right) J\right)=2^{2 m}$.

Proof. We have $\tilde{\Sigma}=C_{m}, R_{1}=A_{2 m+1}$ and $R_{2}=C_{m}$ by (8) of the table in Subsection 4.2. Theorem 4.19 and Example 4.3 imply

$$
\#\left(L_{1} \cap \operatorname{Ad}(a) L_{2}\right)=2^{m}, \quad \#\left(W\left(R_{2}\right) J\right)=2^{2 m} .
$$

Since $J$ is a characteristic element associated with $R_{1}$, there exists $J_{i}(1 \leq i \leq 2 m-1)$ in Example 4.6 such that $J=J_{i}$. Since the multiplicity of any root is equal to 4, (4.5) implies that

$$
8 m^{2}=\operatorname{dim} M=8 \cdot i(m-i) \text {. }
$$


Hence $i=m$. Thus $\#\left(W\left(R_{1}\right) J\right)=\left(\begin{array}{c}2 m \\ m\end{array}\right)$ by Example 4.6.

The following results immediately follows from Theorem 4.19.

Corollary 4.23. Assume that $L_{1} \cap \operatorname{Ad}(a) L_{2}$ is discrete. If $\mathfrak{a}=\mathfrak{a}_{1}$ then $\tilde{\Sigma}=R_{1}$ and $L_{1} \cap \operatorname{Ad}(a) L_{2}=W\left(R_{1}\right) J$.

The following examples satisfy the assumption $\mathfrak{a}=\mathfrak{a}_{1}$ of Corollary 4.23 .

Example 4.24. If

$$
\left(M, L_{1}, L_{2}\right)=(S p(2 m) / U(2 m), S p(m), U(2 m) / O(2 m)),
$$

then $\tilde{\Sigma}=R_{1}=C_{m}, R_{2}=C_{2 m}$ and

$$
\begin{aligned}
& L_{1} \cap \operatorname{Ad}(a) L_{2}=W\left(R_{1}\right) J=W\left(R_{2}\right) J \cap \mathfrak{a}, \\
& \#\left(L_{1} \cap \operatorname{Ad}(a) L_{2}\right)=2^{m}, \quad \#\left(W\left(R_{2}\right) J\right)=2^{2 m} .
\end{aligned}
$$

Proof. We have $\tilde{\Sigma}=R_{1}=C_{m}$ and $R_{2}=C_{2 m}$ by (2) of the table in Subsection 4.2. Theorem 4.19 and Example 4.3 imply \# $\left(L_{1} \cap \operatorname{Ad}(a) L_{2}\right)=$ $2^{m}$ and $\#\left(W\left(R_{2}\right) J\right)=2^{2 m}$.

Example 4.25. If

$$
\left(M, L_{1}, L_{2}\right)=\left(E_{7} / S^{1} \cdot E_{6}, S^{1} \cdot E_{6} / F_{4},(S U(8) / S p(4)) / \mathbb{Z}_{2}\right),
$$

then $\tilde{\Sigma}=R_{1}=C_{3}, R_{2}=E_{7}$ and

$$
\begin{aligned}
& L_{1} \cap \operatorname{Ad}(a) L_{2}=W\left(R_{1}\right) J=W\left(R_{2}\right) J \cap \mathfrak{a}, \\
& \#\left(L_{1} \cap \operatorname{Ad}(a) L_{2}\right)=2^{3}, \quad \#\left(W\left(R_{2}\right) J\right)=2^{3} \cdot 7 .
\end{aligned}
$$

Proof. We can verify $\tilde{\Sigma}, R_{1}$ and $R_{2}$ by (4) of the table in Subsection 4.2. Theorem 4.19 and Example 4.3 imply $\#\left(L_{1} \cap \operatorname{Ad}(a) L_{2}\right)=2^{3}$. Example 4.10 implies \# $\left(W\left(R_{2}\right) J\right)=2^{3} \cdot 7$.

Example 4.26. If

$$
\left(M, L_{1}, L_{2}\right)=\left(G_{n}\left(\mathbb{C}^{2 n}\right), U(n), G_{n}\left(\mathbb{R}^{2 n}\right)\right),
$$

then $\tilde{\Sigma}=R_{1}=C_{n}, R_{2}=A_{2 n-1}$ and

$$
\begin{aligned}
& L_{1} \cap \operatorname{Ad}(a) L_{2}=W\left(R_{1}\right) J=W\left(R_{2}\right) J \cap \mathfrak{a}, \\
& \#\left(L_{1} \cap \operatorname{Ad}(a) L_{2}\right)=2^{n}, \quad \#\left(W\left(R_{2}\right) J\right)=\left(\begin{array}{c}
2 n \\
n
\end{array}\right) .
\end{aligned}
$$


Proof. We can verify $\tilde{\Sigma}, R_{1}$ and $R_{2}$ by (1) of the table in Subsection 4.2. Theorem 4.19 and Example 4.3 imply $\#\left(L_{1} \cap \operatorname{Ad}(a) L_{2}\right)=2^{n}$. Since $J$ is a characteristic element associated with $R_{2}$, there exists $J_{i}$ $(1 \leq i \leq 2 n-1)$ in Example 4.6 such that $J=J_{i}$. Since the multiplicity of any root in $R_{2}$ is equal to 1 , (4.5) implies that

$$
2 n^{2}=\operatorname{dim} M=2(2 n-i) i \text {. }
$$

Hence $i=n$. Thus $\#\left(W\left(R_{2}\right) J\right)=\left(\begin{array}{c}2 n \\ n\end{array}\right)$ by Example 4.6.

Example 4.27. If

$$
\left(M, L_{1}, L_{2}\right)=\left(Q_{r+s+t-2}(\mathbb{C}), S^{r-1, s+t-1}, S^{r+s-1, t-1}\right) \quad(s>0, r<t),
$$

then

$$
\tilde{\Sigma}=R_{1}=B_{1}, \quad R_{2}= \begin{cases}B_{\min \{r+s, t\}} & (r+s \neq t) \\ D_{t} & (r+s=t)\end{cases}
$$

and

$$
\begin{aligned}
& L_{1} \cap \operatorname{Ad}(a) L_{2}=W\left(R_{1}\right) J=W\left(R_{2}\right) J \cap \mathfrak{a}, \\
& \#\left(L_{1} \cap \operatorname{Ad}(a) L_{2}\right)=2 r, \quad \#\left(W\left(R_{2}\right) J\right)=2 \min \{r+s, t\} .
\end{aligned}
$$

Proof. We can verify $\tilde{\Sigma}, R_{1}$ and $R_{2}$ by (5) of the table in Subsection 4.2. Theorem 4.19 and Example 4.2 imply $\#\left(L_{1} \cap \operatorname{Ad}(a) L_{2}\right)=2^{r}$. If $r+s \neq t$, then $\#\left(W\left(R_{2}\right) J\right)=2 \min \{r+s, t\}$ by Example 4.2. If $r+s=t$, then $J=J_{1}$ in Example 4.5, since the multiplicity of any root in $R_{2}$ is equal to 1 . Thus \# $\left(W\left(R_{2}\right) J\right)=2 t$ by Example 4.5 .

Example 4.28. If

$$
\left(M, L_{1}, L_{2}\right)=(S O(4 m) / U(2 m), U(2 m) / S p(m), S O(2 m)),
$$

then $\tilde{\Sigma}=R_{1}=C_{m}, R_{2}=D_{m}$ and

$$
\begin{aligned}
& L_{1} \cap \operatorname{Ad}(a) L_{2}=W\left(R_{1}\right) J=W\left(R_{2}\right) J \cap \mathfrak{a}, \\
& \#\left(L_{1} \cap \operatorname{Ad}(a) L_{2}\right)=2^{m}, \quad \#\left(W\left(R_{2}\right) J\right)=2^{2 m+1} .
\end{aligned}
$$

Proof. We can verify $\tilde{\Sigma}, R_{1}$ and $R_{2}$ by (3) of the table in Subsection 4.2. Theorem 4.19 and Example 4.3 imply $\#\left(L_{1} \cap \operatorname{Ad}(a) L_{2}\right)=2^{m}$. Since the multiplicity of any root in $R_{2}$ is equal to 1 , (4.5) implies that $J=J_{2}$ in Example 4.5. Thus \# $\left(W\left(R_{2}\right) J\right)=2^{2 m+1}$ by Example 4.5.

Examples $4.20, \ldots, 4.28$ exhaust all two real forms $L_{1}$ and $L_{2}$ which are not congruent to each other in an irreducible Hermitian symmetric space $M$ of compact type. For each real form $L$ in $M$ we can determine 
the cardinality of $L \cap \operatorname{Ad}(a) L$ described in Theorem 4.14 by the use of Examples in this section. The result is as follows.

\begin{tabular}{|l|l|l|l|}
\hline$M$ & $L$ & $\#(L \cap \operatorname{Ad}(a) L)$ & Example \\
\hline$G_{k}\left(\mathbb{C}^{n}\right)$ & $G_{k}\left(\mathbb{R}^{n}\right)$ & $\left(\begin{array}{l}n \\
k\end{array}\right)$ & 4.16 \\
\hline$G_{2 k}\left(\mathbb{C}^{2 n}\right)$ & $G_{k}\left(\mathbb{H}^{n}\right)$ & $\left(\begin{array}{l}n \\
k\end{array}\right)$ & 4.21 \\
\hline$G_{n}\left(\mathbb{C}^{2 n}\right)$ & $U(n)$ & $2^{n}$ & 4.26 \\
\hline$Q_{n}(\mathbb{C})$ & $S^{k, n-k}$ & $2 k+2$ & 4.27 \\
\hline$S O(4 n) / U(2 n)$ & $U(2 n) / S p(n)$ & $2^{n}$ & 4.28 \\
\hline$S O(2 n) / U(n)$ & $S O(n)$ & $2^{n-1}$ & 4.17 \\
\hline$S p(2 n) / U(2 n)$ & $S p(n)$ & $2^{n}$ & 4.24 \\
\hline$S p(n) / U(n)$ & $U(n) / O(n)$ & $2^{n}$ & 4.15 \\
\hline$E_{6} / S^{1} \cdot \operatorname{Spin}(10)$ & $G_{2}\left(\mathbb{H}^{4}\right) / \mathbb{Z}_{2}$ & $3^{3}$ & 4.20 \\
\hline$E_{6} / S^{1} \cdot \operatorname{Spin}(10)$ & $F_{4} / \operatorname{Spin}(9)$ & 3 & 4.20 \\
\hline$E_{7} / S^{1} \cdot E_{6}$ & $(S U(8) / S p(4)) / \mathbb{Z}_{2}$ & $2^{3} \cdot 7$ & 4.25 \\
\hline$E_{7} / S^{1} \cdot E_{6}$ & $S^{1} \cdot E_{6} / F_{4}$ & $2^{3}$ & 4.25 \\
\hline
\end{tabular}

We explain how to determine $\#(L \cap \operatorname{Ad}(a) L)$ in one line in the above list. We can similarly determine $\#(L \cap \operatorname{Ad}(a) L)$ in the other lines. In the case where $M=E_{6} / S^{1} \cdot \operatorname{Spin}(10)$ and $L=G_{2}\left(\mathbb{H}^{4}\right) / \mathbb{Z}_{2}$, this real form is described as $L_{2}$ in Example 4.20. Theorem 4.14 and Example 4.20 show that $\#(L \cap \operatorname{Ad}(a) L)=\#\left(W\left(R_{2}\right) J\right)=3^{3}$.

\section{THE FIXED POINT SET AND THE INTERSECTION}

Let $L_{1}=F\left(\tau_{1}, M\right)$ and $L_{2}=F\left(\tau_{2}, M\right)$ be two real forms in an irreducible Hermitian symmetric space $M=\operatorname{Ad}(G) J$ of compact type. Since $\operatorname{Ad}(a) L_{2}=F\left(a \tau_{2} a^{-1}, M\right)$ for $a \in G$, we have

$$
L_{1} \cap \operatorname{Ad}(a) L_{2} \subset F\left(\tau_{1}^{-1} a \tau_{2} a^{-1}, M\right) .
$$

In this section we study the relation between $L_{1} \cap \operatorname{Ad}(a) L_{2}$ and the fixed point set $F\left(\tau_{1}^{-1} a \tau_{2} a^{-1}, M\right)$ of a holomorphic isometry $\tau_{1}^{-1} a \tau_{2} a^{-1}$ when $L_{1} \cap \operatorname{Ad}(a) L_{2}$ is discrete. We may assume that $\tau_{1} \tau_{2}=\tau_{2} \tau_{1}$ and $a$ is in $\exp \mathfrak{a}$. After some preparations we will prove the following theorem.

Theorem 5.1. When $L_{1} \cap \operatorname{Ad}(a) L_{2}$ is discrete, then

$F\left(\tau_{1}^{-1} a \tau_{2} a^{-1}, M\right)=\left(\mathfrak{a} \oplus \mathfrak{f}_{0}\right) \cap M, \quad F\left(\tau_{1}^{-1} a \tau_{2} a^{-1}, M\right) \cap \mathfrak{a}=L_{1} \cap \operatorname{Ad}(a) L_{2}$.

Further

(1) If $\mathfrak{f}_{0}$ is abelian, then

$$
F\left(\tau_{1}^{-1} a \tau_{2} a^{-1}, M\right)=W\left(\mathfrak{g}_{12}\right) J .
$$

(2) If $\mathfrak{f}_{0}=\{0\}$, then

$$
F\left(\tau_{1}^{-1} a \tau_{2} a^{-1}, M\right)=L_{1} \cap \operatorname{Ad}(a) L_{2} .
$$


Lemma 5.2. Set $a=\exp H$ for some $H \in \mathfrak{a}$.

(1) If $L_{1}=L_{2}$, then

$$
F\left(\tau_{1}^{-1} a \tau_{2} a^{-1}, M\right)=\left(\mathfrak{a} \oplus \mathfrak{f}_{0} \oplus \sum_{\substack{\left.\lambda \in R^{+} \\ \lambda, H\right\rangle \in \pi \mathbb{Z}}}\left(\mathfrak{f}_{\lambda} \oplus \mathfrak{p}_{\lambda}\right)\right) \cap M
$$

(2) If $L_{1}$ is not congruent to $L_{2}$, then

$$
\begin{aligned}
& F\left(\tau_{1}^{-1} a \tau_{2} a^{-1}, M\right) \\
& =\left(\mathfrak{a} \oplus \mathfrak{f}_{0} \oplus \sum_{\substack{\lambda \in R^{+} \\
\langle\lambda, H\rangle \in \pi \mathbb{Z}}}\left(\mathfrak{f}_{\lambda} \oplus \mathfrak{p}_{\lambda}\right) \oplus \sum_{\substack{\alpha \in W^{+} \\
\left\langle\alpha, H \in \pi_{2}+\pi \mathbb{Z}\right.}} V_{\alpha}^{\perp}\left(\mathfrak{f}_{1} \cap \mathfrak{p}_{2}\right) \oplus V_{\alpha}^{\perp}\left(\mathfrak{f}_{2} \cap \mathfrak{p}_{1}\right)\right) \cap M .
\end{aligned}
$$

Proof. We only give the proof of (2) since we can prove (1) in a similar manner to the proof of (2).

Since $\theta_{i}=-\tau_{i}$ on $M \subset \mathfrak{g}([16])$, we have

$$
F\left(\tau_{1}^{-1} a \tau_{2} a^{-1}, M\right)=F\left(\operatorname{Ad}\left(a^{-2}\right) \theta_{1} \theta_{2}, M\right) .
$$

Since $\mathfrak{g}=\mathfrak{g}_{12} \oplus\left(\mathfrak{f}_{1} \cap \mathfrak{p}_{2}\right) \oplus\left(\mathfrak{f}_{2} \cap \mathfrak{p}_{1}\right)$, we have

$$
\begin{aligned}
& F\left(\operatorname{Ad}\left(a^{-2}\right) \theta_{1} \theta_{2}, \mathfrak{g}\right) \\
& =F\left(\operatorname{Ad}\left(a^{2}\right), \mathfrak{g}_{12}\right) \oplus F\left(-\operatorname{Ad}\left(a^{2}\right),\left(\mathfrak{f}_{1} \cap \mathfrak{p}_{2}\right) \oplus\left(\mathfrak{f}_{2} \cap \mathfrak{p}_{1}\right)\right) .
\end{aligned}
$$

Lemma 4.12 implies that

$$
F\left(\operatorname{Ad}\left(a^{2}\right), \mathfrak{g}_{12}\right)=\mathfrak{a} \oplus \mathfrak{f}_{0} \oplus \sum_{\substack{\lambda \in R^{+} \\\langle\lambda, H\rangle \in \pi \mathbb{Z}}}\left(\mathfrak{f}_{\lambda} \oplus \mathfrak{p}_{\lambda}\right) .
$$

Lemma 4.13 implies that

$$
\begin{aligned}
& F\left(-\operatorname{Ad}\left(a^{2}\right),\left(\mathfrak{f}_{1} \cap \mathfrak{p}_{2}\right) \oplus\left(\mathfrak{f}_{2} \cap \mathfrak{p}_{1}\right)\right) \\
& =\sum_{\substack{\alpha \in W^{+} \\
\langle\alpha, H\rangle \in \frac{\pi}{2}+\pi \mathbb{Z}}} V_{\alpha}^{\perp}\left(\mathfrak{f}_{1} \cap \mathfrak{p}_{2}\right) \oplus V_{\alpha}^{\perp}\left(\mathfrak{f}_{2} \cap \mathfrak{p}_{1}\right) .
\end{aligned}
$$

Hence we get the assertion.

Proof of Theorem 5.1. The first part follows from Theorems 4.14, 4.18 and Lemma 5.2. In order to prove (1) we assume that $\mathfrak{f}_{0}$ is abelian. Then the maximality of $\mathfrak{a}$ implies that $\mathfrak{a} \oplus \mathfrak{f}_{0}$ is a maximal abelian subalgebra of $\mathfrak{g}_{12}$. Thus (1) holds. (2) is clear from the first part of this theorem. 
In the case where $L_{1}=L_{2}$ we know the structure of $\mathfrak{f}_{0}$ by Tamaru's table $([14])$. In the case where $L_{1}$ is not congruent to $L_{2}$ we know the structure of $\mathfrak{f}_{0}$ by the table in Subsection 4.2.

Example 5.3. We consider a real form $L=U(n)$ in $M=G_{n}\left(\mathbb{C}^{2 n}\right)$. If $L \cap \operatorname{Ad}(a) L$ is discrete, then

$$
L \cap \operatorname{Ad}(a) L=W\left(C_{n}\right) J, \quad F\left(\tau^{-1} a \tau a^{-1}, M\right)=W(\mathfrak{s u}(2 n)) J .
$$

In particular,

$$
\#(L \cap \operatorname{Ad}(a) L)=2^{n}, \quad \# F\left(\tau^{-1} a \tau a^{-1}, M\right)=\left(\begin{array}{c}
2 n \\
n
\end{array}\right) .
$$

\section{REFERENCES}

[1] R. Bott, The geometry and representation theory of compact Lie groups, Representation theory of Lie groups, 1979, 65-90, London Mathematical Society Lecture Note Series 34.

[2] N. Bourbaki, Groupes et algebres de Lie, Hermann, Paris, 1975.

[3] B.-Y. Chen and T. Nagano, A Riemannian geometric invariant and its applications to a problem of Borel and Serre, Trans. Amer. Math. Soc. 308 (1988), $273-297$.

[4] E. Heintze, R. S. Palais, C.-L. Terng and G. Thorbergsson, Hyperpolar actions on symmetric spaces, Geometry, topology, \& physics, 214-245, Conf. Proc. Lecture Notes Geom. Topology, IV, Int. Press, Cambridge, MA, 1995.

[5] S. Helgason, Differential Geometry, Lie groups, and Symmetric spaces, Academic Press, New York, 1978.

[6] O. Ikawa, The geometry of symmetric triad and orbit spaces of Hermann actions, J. Math. Soc. Japan, 63 (2011) 79-136.

[7] O. Ikawa, A note on symmetric triad and Hermann action, Proceedings of the workshop on differential geometry and submanifolds and its related topics, Saga, August 4-6, 2012, 220-229.

[8] O. Ikawa, M. S. Tanaka and H. Tasaki, The fixed point set of a holomorphic isometry and the intersection of two real forms in the complex Grassmann manifold, Springer Proceedings in Mathematics \& Statistics 106, Y.J. Suh et al. (eds.), ICM Satellite Conference on "Real and Complex Submanifolds", (2014), 319-327.

[9] D. P. S. Leung, Reflective submanifolds. IV, Classification of real forms of Hermitian symmetric spaces, J. Differential Geom. 14 (1979), 179-185.

[10] T. Matsuki, Classification of two involutions on compact semisimple Lie groups and root systems, Journal of Lie theory, 12 (2002), 41-68.

[11] M. Takeuchi, On the fundamental group and the group of isometries of a symmetric space, J. Fac. Sci. Univ. Tokyo, Sect. 110 (1964), 88-123.

[12] M. Takeuchi, Stability of certain minimal submanifolds of compact Hermitian symmetric spaces, Tohoku Math. J., (2) 36 (1984), 293-314.

[13] M. Takeuchi, Two-number of symmetric $R$-spaces, Nagoya Math. J., 115 (1989), 43-46. 
[14] H. Tamaru, The local orbit types of symmetric spaces under the action of the isotropy subgroups, Differential geometry and its applications, 11 (1999), 29-38.

[15] M. S. Tanaka and H. Tasaki, The intersection of two real forms in Hermitian symmetric spaces of compact type, J. Math. Soc. Japan 64 (2012), 1297-1332.

[16] M. S. Tanaka and H. Tasaki, Antipodal sets of symmetric $R$-spaces, Osaka J. Math. 50 (2013), 161-169.

[17] M. S. Tanaka and H. Tasaki, The intersection of two real forms in Hermitian symmetric spaces of compact type II, J. Math. Soc. Japan 67 (2015), 275-291.

[18] M. S. Tanaka and H. Tasaki, Correction to: "The intersection of two real forms in Hermitian symmetric spaces of compact type", to appear in J. Math. Soc. Japan.

Department of Mathematics and Physical Sciences, Faculty of Arts and Sciences, Kyoto Institute of Technology, Sakyoku, Kyoto, 6068585 JAPAN

E-mail address: ikawa@kit.ac.jp

Faculty of Science and Technology, Tokyo University of Science, NoDA, CHIBA, 278-8510 JAPAN

E-mail address: tanakamakiko@ma.noda.tus.ac.jp

Division of Mathematics, Faculty of Pure and Applied Sciences, UniVERSITY OF TSUKUBA, TSUKUBA, IBARAKI, 305-8571 JAPAN

E-mail address: tasaki@math.tsukuba.ac.jp 